\title{
LONGITUDINAL MULTI-TRAIT-STATE-METHOD MODEL USING ORDINAL DATA
}

\author{
Richard Shane Hutton
}

A thesis submitted to the faculty of the University of North Carolina at Chapel Hill in partial fulfillment of the requirements for the degree of Master of Arts in the Department of Psychology (Quantitative).

Chapel Hill

2012

Approved by

Sy-Miin Chow, Ph.D.

Robert MacCallum, Ph.D.

A.T. Panter, Ph.D.

David Thissen, Ph.D. 
(C) 2012

Richard Shane Hutton

ALL RIGHTS RESERVED 


\section{ABSTRACT \\ RICHARD SHANE HUTTON: Longitudinal Multi-Trait-State-Method Model using Ordinal Data \\ (Under the direction of Sy-Miin Chow)}

Multi-trait multi-method (MTMM) models were designed as a way to assess convergent and discriminant validity using multiple traits measured by multiple methods. In recent years, longitudinal extensions of multi-trait multi-method models have been proposed in the structural equation modeling framework to evaluate whether and how the trait as well as method factors change over time. This thesis is intended to propose a longitudinal second-order multi-trait-state-method (M-TSM) model that combines a measurement model for ordinal data, a vector autoregressive moving average (VARMA) model at the latent level to examine changes in the state as well as method factors over time, and a second-order factor analytic model to capture shared trait variances among the state affect factors. A set of affect items from the Affective Dynamics and Individual Differences (Emotions and Dynamic Systems Laboratory, 2010) study was used to illustrate the proposed longitudinal M-TSM model. This study features affect items asked in both a lab and a diary setting. Results of the final model showed that the method factors, lab and diary, had perfect stability across time. Furthermore, factor loadings within the lab factor showed a systemic pattern of positive and negative loadings illustrating the complexity of individuals' affective responses to stimuli in the lab setting. Contrary to conventional theories on emotions, the covariances between positive and negative affect factors at the state level were not significantly different from zero; however, evaluation of higher-order shared covariance between the affect factors across method settings indicates a negative association 
between the two at the "trait" level. Comparisons of the auto- and cross-regression regression parameters associated with the state affect factors indicated some key differences in how people regulated negative emotions in a lab versus in a diary setting. Methodological issues associated with fitting the M-TSM model are discussed. 


\section{TABLE OF CONTENTS}

List of Tables $\ldots \ldots \ldots \ldots \ldots \ldots \ldots \ldots \ldots \ldots$ vii

List of Figures $\ldots \ldots \ldots \ldots \ldots \ldots \ldots \ldots \ldots \ldots \ldots \ldots \ldots \ldots$

\section{CHAPTER}

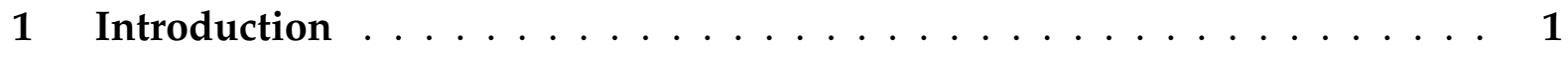

1.1 Multi-Trait Multi-Method $(\mathrm{MTMM}) \ldots \ldots \ldots 2$

1.1.1 Multi-Trait Multi-Method Matrix . . . . . . . . . . . . . 3

1.2 Structural Equation Modeling $(\mathrm{SEM}) \ldots \ldots \ldots$

1.2.1 Multi-Trait Multi-Method SEM Models . . . . . . . . . . . . 7

1.2.2 Ordinal Measurement Model . . . . . . . . . . . . . . . . 10

1.3 Item Response Theory $\ldots \ldots$. . . . . . . . . . . . . . . . 12

1.4 Longitudinal Models . . . . . . . . . . . . . . . . . . . . . 14

1.4.1 Longitudinal Multi-Trait Multi-Method . . . . . . . . . . . . 14

1.4 .2 Time Series Analysis . . . . . . . . . . . . . . . . 15

1.4 .3 Invariance . . . . . . . . . . . . . . . . 17

1.5 Objectives for Thesis $\ldots \ldots \ldots \ldots$

2 Methods ......................... 20

2.1 Illustrative Example . . . . . . . . . . . . . . . . . 20

2.1.1 Participants and Measures . . . . . . . . . . . . . 20

2.1 .2 Procedures . . . . . . . . . . . . . . . . . . 21

2.2 Model Descriptions . . . . . . . . . . . . . . . . . . . . . . 22

2.2.1 Preliminary Data Exploration . . . . . . . . . . . . 22 
2.2.2 Item Exploratory Analysis . . . . . . . . . . . . . . . . . 22

2.2.3 Longitudinal M-TSM Model for the PANAS Data . . . . . . . 23

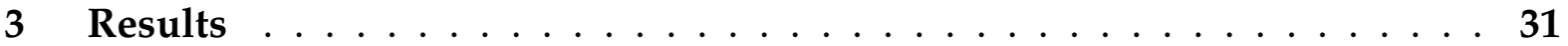

3.1 Results for Item Analysis . . . . . . . . . . . . . . . . . . 31

3.1 .1 IRT Analyses . . . . . . . . . . . . . . . . . . . . . 31

3.1.2 Establishing Longitudinal Invariance . . . . . . . . . . . . 32

3.2 Most Parsimonious Model . . . . . . . . . . . . . . . . . . . 33

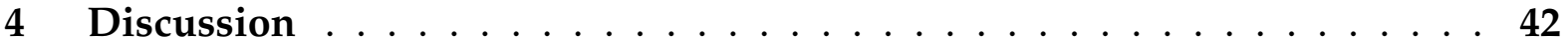

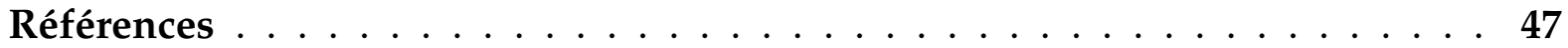




\section{LIST OF TABLES}

2.1 Models Used to Examine Longitudinal Invariance in the PANAS Items. . . 28

3.1 Final List of PANAS Items Retained. . . . . . . . . . . . . . . . . 32

3.2 Models Used to Examine Perfect Stability in the Method Factors . . . . . . 35

3.3 Factor Loadings for the Most Parsimonious Model . . . . . . . . . . 36

3.4 Random Shock Variance Estimates for the Most Parsimonious Model . . . 39

3.5 Time Series Parameters for the Most Parsimonious Model . . . . . . . . . . 39 


\section{LIST OF FIGURES}

1.1 Synthetic multitrait-multimethod matrix taken from Campbell and Fiske (1959), pg. 82. . . . . . . . . . . . . . . . . . . . 5

1.2 Example of a SEM-MTMM model. . . . . . . . . . . . . . . . . 8

1.3 Example of a correlated trait-correlated method model. . . . . . . . . . . . 9

1.4 Example of a correlated trait-correlated uniqueness model. . . . . . . . . . 10

1.5 Example of a correlated trait-correlated method minus one model. . . . . . 11

2.1 Example of (a) a"more desirable" item and (b) a "less desirable" item. . . . 23

2.2 Path diagram of a longitudinal M-TSM model with VAR(1) relationships at the factor level. . . . . . . . . . . . . . . . . . . 27

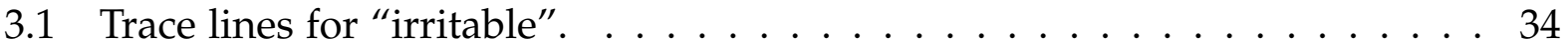

3.2 Simulated trajectories of lag-two AR processes. . . . . . . . . . . . . 40 


\section{CHAPTER 1}

\section{Introduction}

Multi-trait multi-method (MTMM) models have long been used by behavioral and social scientists to study different traits assessed by different methods (e.g., Winne \& Marx, 1977; Waksman, 1978; Andrews, 1984; Bagozzi \& Yi, 1991; Luzzo, 1993). Longitudinal MTMM models provide one possible way for researchers to study how traits assessed by various methods vary over time. Such models not only allow researchers to examine whether or not traits are changing over time, but also provide a proxy for studying the stability of the assessment methods over time. Stability is a key feature that distinguishes trait processes from state processes. Trait processes refer to stable constructs that remain relatively constant over time; however, state processes refer to volatile constructs that change on a moment-to-moment basis (Nesselroade \& Bartsch, 1977).

With ecological momentary assessment designs such as experience sampling and daily diary designs becoming increasingly popular (Iida, Shrout, Laurenceau, \& Bolger, in press), it is important to develop a longitudinal model that is suited for representing the kind of less structured, "state-like" changes often seen in such data. While longitudinal variations of MTMM models have been proposed by other researchers, the change processes assumed in such models are typically structured growth processes that are relatively smooth Grimm, Pianta, \& Konold, 2009; Cole, Martin, Powers, \& Trugilo, 1996; Geiser, Eid, Nussbeck, Courvoisier, \& Cole, 2010). 
Ecological momentary assessment data, in contrast, are often characterized by less systematic ebbs and flows driven by external shocks or events.

The purpose of this thesis is to propose a longitudinal second-order MTMM model that combines a vector autoregressive moving average (VARMA) model at the latent level and a measurement model for ordinal data to represent relatively volatile change processes over time. The VARMA model included in the proposed longitudinal MTMM model is especially suited for representing fluctuations in state processes. In this way, the proposed model is a multi-"state" multi-method longitudinal model at the first-order level and will be referred to as a multi-trait-state-method model (M-TSM). Another major issue the proposed model serves to address is how MTMM models may be adapted for use with ordinal data. Likert-type items are relatively common in social and behavioral sciences. Proper modeling techniques are needed to handle the ordinal nature of this type of data.

The introduction is organized as follows. I first introduce the MTMM design and define construct validity followed by a discussion of the original MTMM matrix. Next, structural equation modeling (SEM) - the modeling framework in which the proposed longitudinal M-TSM model is structured-as well as various SEM-MTMM models are introduced. In addition, a discussion of the ordinal measurement model is given. I then present an introduction to item response theory, particularly the graded response model, which is used to evaluate the psychometric properties of a set of items to be used for model fitting purposes. Finally, an overview of the VARMA model is given followed by a discussion of the importance of longitudinal measurement invariance.

\subsection{Multi-Trait Multi-Method (MTMM)}

A MTMM design consists of the measurement of at least two traits using at least two assessment methods (Campbell \& Fiske, 1959). Not only are multiple methods of assessment common in the social sciences (e.g., parent vs. teacher ratings), so is the 
need to understand whether or not these methods measure what they are intended to measure. As such, violations of construct validity constitute a source of confound in scientific studies. That is, researchers have the task of determining if their measures are adequately representing the construct of interest.

In discussing MTMM, it is useful to first review the concepts of convergent, discriminant and construct validity. Construct validity answers the question: are we measuring what we intend to measure? That is, is there an association between the measures and the theoretical construct they are purported to measure? Furthermore, construct validity can be decomposed into two subparts: convergent and discriminant validity. Convergent validity helps determine whether or not the measures that are supposed to be related actually are. Conversely, discriminant validity helps determine whether or not the measures that are not supposed to be related actually are not.

\subsubsection{Multi-Trait Multi-Method Matrix}

For decades, the MTMM Matrix has been used to examine convergent, discriminant and construct validity. The MTMM Matrix is a correlation matrix that represents the intercorrelations between trait and method factors. Figure 1.1 shows a classic MTMM matrix taken from Campbell et Fiske (1959). This synthetic matrix is a correlation matrix composed of the correlations among three different traits measured using three different methods. While all of the off-diagonal elements represent correlations, each of the diagonal elements of the MTMM matrix consists of the reliability estimate of one particular measure. The solid triangles represent the heterotrait-monomethod triangles and the dashed triangles represent the heterotraitheteromethod triangles. The heterotrait-monomethod triangles represent correlations that share the same measurement method. Conversely, the heterotrait-heteromethod triangles represent correlations that do not share the same measurement method. The diagonals between the heterotrait-heteromethod triangles are called validity di- 
agonals. These diagonals represent the correlations between measures of the same trait but assessed using different methods.

The validity diagonal contains information about convergent validity. That is, high correlations between measures of the same trait would be expected even if different methods are being used. For example, in Figure 1.1 the correlations along the validity diagonal are relatively high in comparison with the neighboring correlations. The neighboring correlations in the heterotrait-heteromethod triangles represent the correlations between different traits and methods. This provides information about discriminant validity; namely, these measures should have low correlations because they are not related. Finally, the heterotrait-heteromethod triangles allow for the examination of a method effect. That is, if relatively high correlations exist within these triangles, then the methods are having a strong influence since none of these correlations share the same trait.

The MTMM matrix has several limitations; one major limitation lies in the difficulties for researchers to quantify and infer the existence of construct validity simply by eyeballing the correlations. That is, there is no set standard to help gauge whether the different kinds of validity are attained. It is also impossible to separate method and error variances, which may lead to spurious correlations and wrong conclusions (Schmitt \& Stults, 1986). Finally, the MTMM matrix requires the measures to be conditionally independent once the shared variances attributable to common method and common trait have been accounted for (Campbell \& Fiske, 1959); whereas, in reality correlations might exist between method factors and trait factors. Given these limitations of the MTMM matrix, many researchers have turned to a statistical technique called structural equation modeling (SEM) as an alternative method for examining construct validity. 


\begin{tabular}{|c|c|c|c|c|c|c|c|c|c|c|}
\hline & \multirow[b]{2}{*}{ Traits } & \multicolumn{3}{|c|}{ Method 1} & \multicolumn{3}{|c|}{ Method 2} & \multicolumn{3}{|c|}{ Method 3} \\
\hline & & $A_{1}$ & $\mathrm{~B}_{1}$ & $\mathrm{C}_{1}$ & $\mathrm{~A}_{2}$ & $\mathrm{~B}_{2}$ & $\mathrm{C}_{2}$ & $A_{8}$ & $\mathrm{~B}_{3}$ & $C_{3}$ \\
\hline \multirow{3}{*}{ Method 1} & $A_{1}$ & $(.89)$ & & & & & & & & \\
\hline & $B_{1}$ & .51 & & & & & & & & \\
\hline & $C_{1}$ & .38 & & 76) & & & & & & \\
\hline \multirow{3}{*}{ Methorl 2} & $A_{2}$ & .57 & & 09 & $(.93)$ & & & & & \\
\hline & $\mathrm{Ba}_{2}$ & & & & .68 & 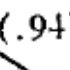 & & & & \\
\hline & $\mathrm{C}_{2}$ & .11 & & .46 & .59 & & $(.84)$ & & & \\
\hline \multirow{3}{*}{ Method 3} & $\mathrm{~A}_{3}$ & .56 & & $\overline{11 !}$ & .67 & & $.3 \overline{4}$ & $(.94)$ & & \\
\hline & $\mathrm{B}_{3}$ & & & & & & 34 & .67 & 10 & \\
\hline & $\mathrm{C}_{3}$ & .11 & & & 1.34 & & .58 & .58 & & $.85)$ \\
\hline
\end{tabular}

Figure 1.1: Synthetic multitrait-multimethod matrix taken from Campbell and Fiske (1959), pg. 82. 


\subsection{Structural Equation Modeling (SEM)}

SEM is a set of statistical techniques that allows for the evaluation of relationships among variables, specifically, the relationships between observed variables (usually called manifest variables) and unobserved latent variables. SEM is also known as covariance structure modeling because some of the earliest structural equation models focus explicitly on representing the covariance structures among measured and latent variables (Jöreskog, 1973, 1974a). In order to describe these relationships, two models are defined: 1) the measurement model and 2) the structural model. The measurement model relates the manifest variables to the latent variables; whereas, the structural model relates the latent variables to each other.

The measurement model consists of two equations. One equation links manifest variables and exogenous latent variables and a second equation links manifest variables and endogenous latent variables. The first measurement equation is defined as

$$
\boldsymbol{x}_{i t}=\boldsymbol{v}_{x}+\boldsymbol{\Lambda}_{x} \boldsymbol{\xi}_{i}+\boldsymbol{\delta}_{i}
$$

where $x_{i t}$ is a $q \times 1$ vector of manifest variables for person $i, v$ is a $q \times 1$ vector of intercepts, $\boldsymbol{\Lambda}_{x}$ is a $q \times n$ factor loading matrix, $\boldsymbol{\xi}_{i}$ is an $n \times 1$ vector of latent exogenous variables for person $i$, and $\delta_{i}$ is a $q \times 1$ vector of measurement errors. It is assumed that $\delta_{i}$ is normally distributed, $\xi_{i}$ and $\delta_{i}$ are uncorrelated and both have expected values of zeroes.

The second measurement equation is defined as

$$
\boldsymbol{y}_{i}=\boldsymbol{v}_{y}+\boldsymbol{\Lambda}_{y} \eta_{i}+\boldsymbol{\varepsilon}_{i}
$$

where $\boldsymbol{y}_{i}$ is a $p \times 1$ vector of manifest variables for person i, $\boldsymbol{v}_{y}$ is a $p \times 1$ vector

of intercepts, $\boldsymbol{\Lambda}_{y}$ is a $p \times m$ factor loading matrix, $\boldsymbol{\eta}_{i}$ is an $m \times 1$ vector of latent 
endogenous variables for person $i$, and $\varepsilon_{i}$ is a $p \times 1$ vector of measurement errors. Once again, it is assumed that $\varepsilon_{i}$ is multivariate normally distributed with a vector of zeroes as expected values and covariance matrix, $\boldsymbol{\Psi}$. In addition, $\boldsymbol{\eta}_{i}$ and $\boldsymbol{\varepsilon}_{i}$ are assumed to be uncorrelated.

Finally, the structural model is defined as

$$
\eta_{i}=\alpha+\mathbf{B} \eta_{i}+\Gamma \xi_{i}+\zeta_{i}
$$

where $\boldsymbol{\alpha}$ is a $m \times 1$ vector of intercepts, $\boldsymbol{B}$ is an $m \times m$ matrix linking the latent endogenous variables, $\Gamma$ is an $m \times n$ matrix linking the latent exogenous variables to the latent endogenous variables and $\zeta_{i}$ is a $m \times 1$ vector of residual errors which is assumed to be multivariate normally distributed with zero means and covariance matrix $\Psi$.

\subsubsection{Multi-Trait Multi-Method SEM Models}

Thus far, I have introduced the MTMM matrix developed by Campbell and Fiske (1959) and provided a brief introduction to the SEM framework. Next, I will review how the MTMM design and how pertinent validity-related questions can be formulated as part of a structural equation model. Central to the SEM-MTMM framework is a set of latent variables representing traits and a second set of latent variables representing methods. Each manifest variable loads onto one trait and one method. Figure 1.2 shows an example of a basic SEM-MTMM. Note that this model consists of only endogenous latent variables; that is, there is no exogenous component. In addition, the trait and method factors are uncorrelated with one another. Over the years, several different SEM-MTMM models have been formulated. I will provide a quick review of the primary models.

The Correlated Trait-Correlated Method Model. The correlated trait-correlated method model (Jöreskog, 1974b; Widaman, 1985) is an SEM model that most closely 


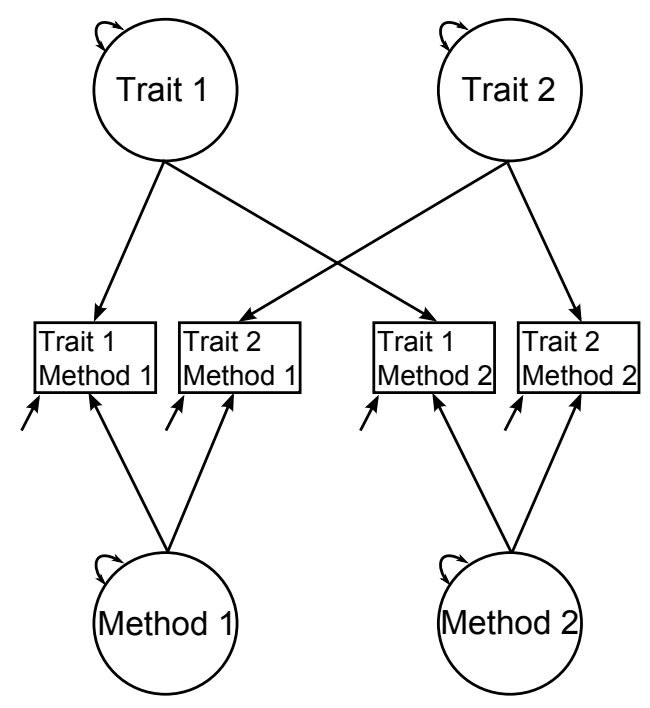

Figure 1.2: Example of a SEM-MTMM model.

resembles the original MTMM matrix validity defined by Campbell and Fiske (1959) due to its formulation and interpretation; an example is shown in Figure 1.3. This model has a latent variable for each trait and each method. Furthermore, correlations exist between the trait factors and sometimes also the method factors; however, there is no correlation between method and trait factors. One major benefit of this model is the ease of interpretation. Large trait factor loadings represent convergent validity and small correlations between trait factors represent discriminant validity. Thus, validity is defined in much the same ways as how Campbell and Fiske (1959) originally defined validity. The major disadvantage of these models lies in problems with identification and convergence (Marsh, 1989; Marsh \& Bailey, 1991); issues include (1) underidentified models, (2) negative variances and (3) correlations outside of the proper range. While other models are not immune from these issues, they are most prevalent in the correlated trait-correlated method model.

The Correlated Trait-Correlated Uniqueness Model. The correlated trait-correlated uniqueness model (Kenny, 1976; Marsh, 1989) is much like the correlated trait- 


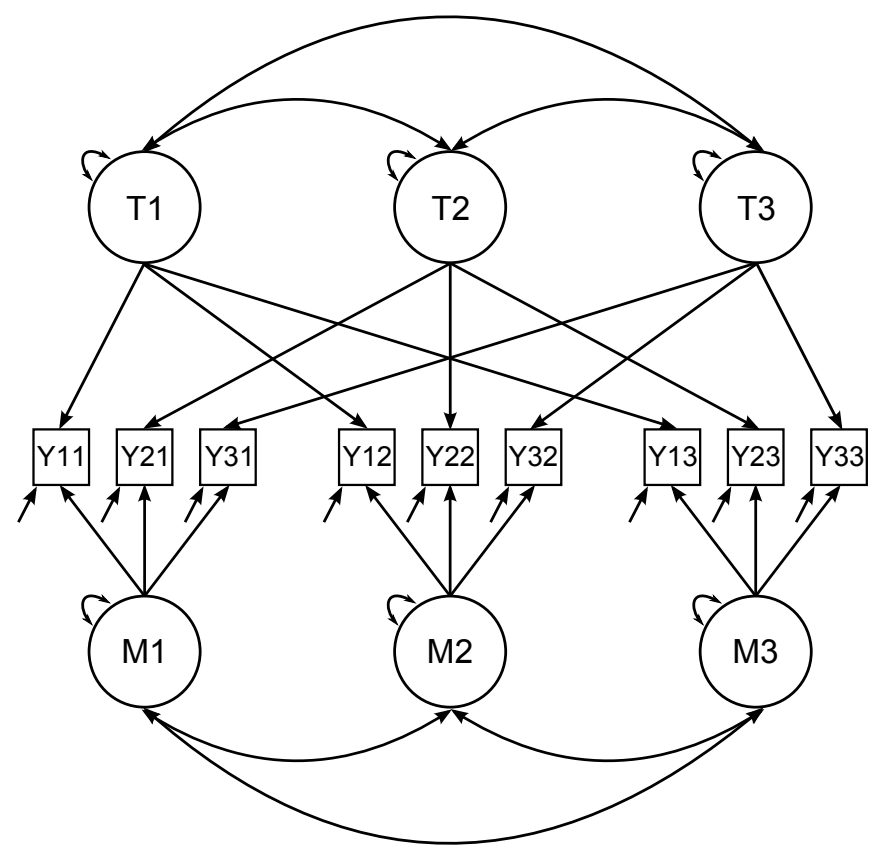

Figure 1.3: Example of a correlated trait-correlated method model.

correlated method model but method factors are not specified explicitly (see Figure 1.4). Instead, to account for the method effects, error terms are allowed to be correlated within methods, with no correlations allowed between the errors of manifest variables measured using different methods. Not allowing for correlations between the errors of different methods is equivalent to assuming that the methods factors in Figure 1.3 are independent, which may be overly restrictive in some applications. Furthermore, another limitation is that the error terms represent both measurement errors and the residual effects that are idiosyncractic to each method; it is not possible to decompose the two. This model does have the advantage of fewer identification problems than the correlated trait-correlated method model proposed by Widaman (1985).

The Correlated Trait-Correlated Method Minus One Model. The correlated traitcorrelated method minus one model (Eid, 2000) is similar to the correlated trait- 


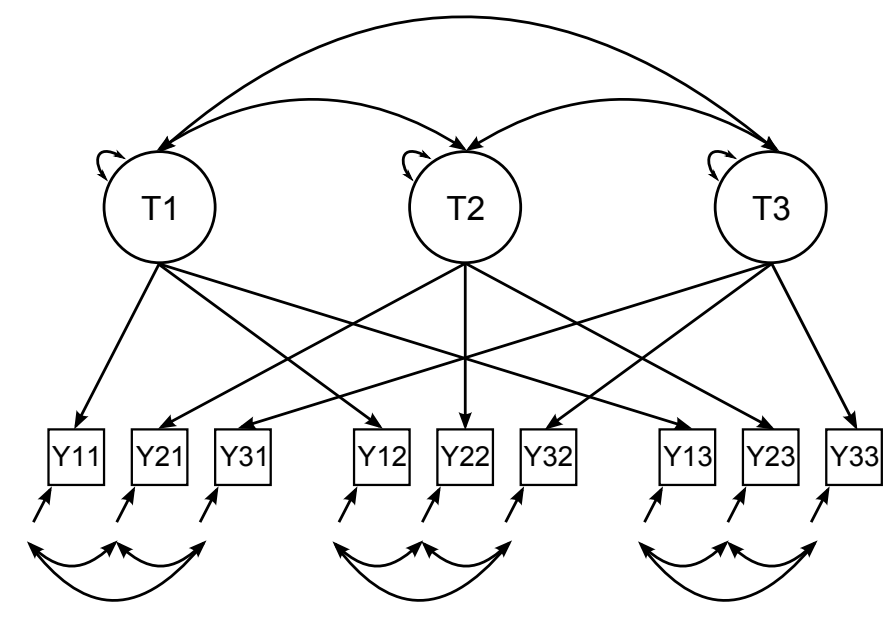

Figure 1.4: Example of a correlated trait-correlated uniqueness model.

correlated method model (Jöreskog, 1974b; Widaman, 1985), except that one method serves as a reference method (see Figure 1.5). This latter model is nested within the correlated-trait correlated-method model, with special constraints included in the factor loading matrix. That is, the loadings of the manifest measures on one of the method factors - namely, the reference method - are fixed at zeroes. This implies that there are no shared sources of variance among the manifest variables measured using the reference method after the shared variance due to trait has been extracted. In addition, the loadings of the trait factors on manifest variables measured using the reference method are set to one as added identification constraints. The major advantage of this model is that by imposing more identification constraints than the standard correlated-trait correlated-method model, convergence issues are reduced; however, it can be difficult to determine which method to use as the reference method.

\subsubsection{Ordinal Measurement Model}

The measurement model shown in Equations $1.1 \& 1.2$ assumes that continuous indicators are used to identify the latent variables; therefore, using ordinal indica- 


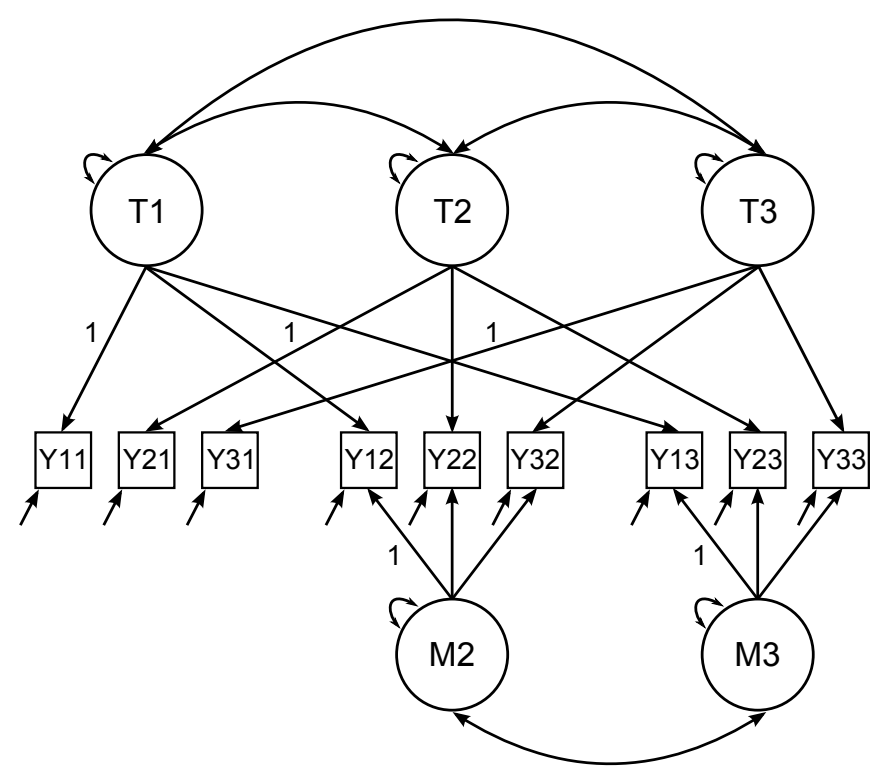

Figure 1.5: Example of a correlated trait-correlated method minus one model.

tors requires a different measurement model to be specified. One common way of incorporating ordinal manifest variables is to use an ordered probit link. The basic assumption made is that responses to the ordinal observed measured variables $y$ are influenced by an unobserved, continuous measured variable $y^{*}$. Therefore, only response categories are observed. That is, for ordered response categories $k=1,2, \ldots, m$, thresholds are defined as

$$
y_{i, j}=k \text { if } \tau_{j, k-1}<y^{*} \leq \tau_{j, k}
$$

where $\tau_{j, k}$ are threshold parameters for the $j$ th items. The thresholds represent cutpoints on the continuous latent variable $y^{*}$ that determine whether respondents endorse a particular category $k$ on the $j$ th observed item $y$. When a probit link is specified, the cumulative distribution function (CDF) of $y^{*}$ is assumed to be normal. It is important to note that other CDF's can be used, which will be shown in the next 
section. When estimating ordered categorical indicators in SEM, error terms for the indicators are not being estimated; instead, the threshold parameters, $\tau_{j, k}$, are estimated. Furthermore, treating the ordered categorical items as continuous would not be appropriate and could lead to spurious results (DiStefano, 2002). Various estimation methods for handling categorical data in SEM have been summarized by Wirth and Edwards (2007).

\subsection{Item Response Theory}

Item Response Theory (IRT) is a set of statistical models that relates the probability of an individual's response on an item as a function of item parameters and an underlying latent trait (Lord, 1980). In contrast to the SEM framework presented above, commonly used IRT models assume a logit link for $y$; that is, IRT uses a logistic distribution function instead of a normal distribution function (probit) as the $\mathrm{CDF}$ for the continuous latent variable, $y^{*}$. One important advantage of using IRT is that the various estimation procedures and associated software packages have been developed specifically to handle large-scale categorical data sets. Thus, the assess-

ment of psychometric properties of categorical items, including ordinal items, can be performed in a computationally efficient manner.

In the IRT framework, two item-related parameters are typically of primary interest: discrimination and difficulty parameters. Discrimination represents how related an item is to the latent trait; whereas, difficulty represents the trait level needed so that there is a 0.5 probability of endorsing a particular item (in the case of binary items) or response category (in the case of ordinal items). An item that is highly discriminating provides more information about the latent construct than a item that is less discriminating. A one-parameter IRT model estimates the difficulty parameter for each item; whereas, a two-parameter model estimates both difficulty and discrimination.

A particular IRT model can be chosen depending on the needs of the researcher 
and the type of data used. For ordinal data, one commonly used model is the graded response model (Samejima, 1969). The graded response model is a two-parameter model and it postulates that the probability for individual $i$ to endorse the $k$ th or other higher response categories $(k=1,2, \ldots, m)$, is defined as

$$
P\left(y_{i j} \geq k \mid \theta_{i}, a_{j}, b_{j, k}\right)=P_{j, k}\left(\theta_{i}\right)=\frac{1}{1+\exp \left[-a_{j}\left(\theta_{i}-b_{j, k}\right)\right]}
$$

where the equation represents the probability of responding in category $k$ or higher given $\theta$, the individual's latent trait level. The parameter $a_{j}$ is the discrimination parameter for item $j$ and $b_{j, k}$ is the $k$ th difficulty parameter for the $j$ th item. In other words, Equation 1.5 provides a probabilistic representation of responding in a particular category or higher given a certain level of $\theta$.

A graphical representation of the differences between the conditional response probabilities of adjacent response categories are known as trace lines (also called item characteristic curves). That is, a trace line is the difference between the probabilities of two adjoining categories, corresponding to $P\left(y_{i j}=k \mid \theta_{i}, a_{j}, b_{j, k}\right)$. Mathematically, a trace line, $T_{j}$, for item $\mathrm{j}$ and category $\mathrm{k}$ are represented as

$$
\begin{aligned}
T_{j} & =\frac{1}{1+\exp \left[-a_{j}\left(\theta_{i}-b_{j, k-1}\right)\right]}-\frac{1}{1+\exp \left[-a_{j}\left(\theta_{i}-b_{j, k}\right)\right]} \\
& =T_{j}^{*}(k)-T_{j}^{*}(k+1)
\end{aligned}
$$

Additionally, information curves help to determine how much information an item gives us to estimate different levels of $\theta$. Information determines at what levels of $\theta$ an item is best at discriminating between individuals. That is, higher information allows for more precision in measuring $\theta$. It is important to note that IRT and the ordinal measurement model in the SEM framework are mathematically equivalent (Wirth \& Edwards, 2007; Takane \& de Leeuw, 1987). The relationship can be 
expressed as

$$
a_{j}=\left(\frac{\lambda_{j}}{\sqrt{1-\lambda_{j}^{2}}}\right) D \text { and } b_{j, k}=\frac{\tau_{j, k}}{\lambda_{j}}
$$

where $\lambda_{i}$ is the factor loading ${ }^{1}$ for the $j$ th item, $\mathrm{D}=1.7$ is the scaling factor, and $\tau_{j, k}$ is the threshold for the $j$ th item.

Finally, in order to properly estimate the item parameters, IRT models require a set of locally independent items (i.e no local dependence). That is, item responses are independent from each other controlling for trait level. There are multiple statistics to evaluate local dependence; the $\chi^{2}$ LD statistic (Chen \& Thissen, 1997) will be used. The $\chi^{2}$ LD statistic examines the covaration between observed and expected frequencies between pairs of items. This covariation should be zero if there is no local dependence; however, if this covaration differs significantly then there exists local dependence between a pair of items (i.e. violation of local independence).

\subsection{Longitudinal Models}

\subsubsection{Longitudinal Multi-Trait Multi-Method}

The idea of using MTMM longitudinally was first introduced by Werts, Jöreskog and Linn (1972) to study growth. Since then, several longitudinal MTMM models have been proposed to date (Conley, 1985; Cudeck, 1985; Burns, Walsh, \& Gomez, 2003; Marsh \& Grayson, 1995; Scherpenzeel \& Saris, 2007). This section is intended to briefly summarize the various longitudinal MTMM models that have been proposed within the SEM framework.

Grimm et al. (2009) presented a longitudinal version of the correlated traitcorrelated method model wherein changes in the trait factors over time are represented using second-order latent growth curve models. In addition to using growth curve models to examine linear changes in the trait factors, they also tested the in-

\footnotetext{
${ }^{1}$ IRT models assume unidimensionality so a factor index is typically not used
} 
variance of the repeated measures over time. The authors noted several convergence issues with their model especially when the correlations are high among factors. Furthermore, the authors suggested building the model at each time point prior to incorporating all repeated measures to evaluate trends or changes over time to aid estimation difficulties.

Cole et al. (1996) formulated a longitudinal correlated trait-correlated uniqueness model to study the relationship between social and academic competence and depression using two waves of data. In line with properties of the cross-sectional correlated trait-correlated uniqueness model (Kenny, 1976; Marsh, 1989), errors of the same measures were allowed to be correlated across as well as within waves. The authors also used correlations to examine stability across waves of data using a test-retest type of design. Finally, Geiser et al. (2010) presented a longitudinal version of the correlated trait-correlated method minus one model in which the latent difference model (McArdle \& Hamagami, 2001b) was used to represent changes in the trait factors over time.

The longitudinal MTMM models reviewed above are similar in that the correlations between trait factors over time are hypothesized to stem from some forms of structured growth curve processes ${ }^{2}$; however, none of the models employ time series techniques. Furthermore, the proposed model uses three waves of data, which is more beneficial than two waves of data typically used in the aforementioned models (Willett, 1989).

\subsubsection{Time Series Analysis}

Time series analysis is a set of statistical methods for analyzing time series data, namely, data that are collected over time. Two commonly used univariate time series models are autoregressive and moving average processes (Chatfield, 2004; Wei, 1990;

\footnotetext{
${ }^{2}$ The latent difference score models proposed by McArdle and colleagues (2001b) can be regarded as constrained versions of the latent growth curve models
} 
Hamilton, 1994). Time series models are traditionally used with single-subject time series data with many repeated measurement occasions. Thus, the subject index is typically omitted from the corresponding models. Here, these models are used with multiple-subject longitudinal panel data with many participants and relatively few time points. I therefore include a subject index in all the equations that follow.

Mathematically, an autoregressive model of lag order $r$, denoted as $\operatorname{AR}(r)$, for a measured variable, $y_{i t}$, of person $i$ at time $t$, is defined as

$$
y_{i t}=\sum_{u=1}^{r} \varphi_{u} y_{i, t-u}+\varepsilon_{i t}
$$

where $\varphi_{u}(u=1, \ldots, r)$ are autoregressive parameters and $\varepsilon_{i t}$ is an error term. Browne and Nesselroade (2005) referred to these "errors" as random shocks because they can be regarded as shocks whose impact continues to affect later $y_{i t}$. In the case of the AR process, the lagged effects of such shocks are transmitted through previous $y_{i t}$. The error term $\varepsilon_{i t}$ has the following assumptions: zero mean, variance $\sigma^{2}$, and uncorrelated for different time values. Furthermore, $r$ is the highest lag order of the process and the AR processes is considered to be weakly stationary if $E\left[y_{i t}\right]$ and $\operatorname{Var}\left[y_{i t}\right]$ are constant (Chatfield, 2004)

In a similar vein, a moving average model of lag order $s$, denoted as MA(s), for a measured variable $y_{i t}$, is defined as

$$
y_{i t}=\sum_{v=1}^{s} \delta_{v} \varepsilon_{i, t-v}+\varepsilon_{i t}
$$

where $\delta_{v}$ are moving average parameters and $\varepsilon_{i t}$ is an error term or random shock. A moving average process is considered to be invertible if it can be rewritten as a linear combination of its past values (Chatfield, 2004). Both equations 1.8 and 1.9 can be combined to yield an auto-regressive moving-average (ARMA) model of order $(r, s)$.

The vector auto-regressive moving-average (VARMA) is a multivariate extension 
of the univariate $\mathrm{AR}(\mathrm{r})$ and $\mathrm{MA}(\mathrm{s})$ models shown in Equations 1.8 and 1.9. The VARMA model can be structured within the SEM framework wherein a series of autoregressive and moving average components are represented in the structural equation. For example, combining Equations 1.8 and 1.9 the VARMA(r,s) model is obtained

$$
\boldsymbol{y}_{i t}=\sum_{u=1}^{r} \varphi_{u} \boldsymbol{y}_{i, t-u}+\sum_{v=1}^{s} \delta_{v} \boldsymbol{\varepsilon}_{i, t-v}+\boldsymbol{\varepsilon}_{i t}
$$

where $y_{i t}$ is now a vector of manifest variables and $\varepsilon_{i t}$ is the corresponding vector of random shocks. Multiple researchers have evaluated variations of univariate and multivariate time series models in the context of SEM, (e.g., McArdle \& Hamagami, 2001a; Bollen \& Curran, 2004), but no work has been done combining SEM-MTMM models with time series analysis. Not only do autoregressive parameters allow for the examination of the influence of one time point on another, they offer a straightforward index for evaluating the stability of a construct of interest over time. In addition, cross-regression parameters allow researchers to examine the influence of past constructs on other constructs of interest. More importantly, times series models are suited for representing less structured change processes typically seen in state fluctuations, such as those evidenced in emotions data.

\subsubsection{Invariance}

In the study of longitudinal change processes, it is useful to understand whether or not parameter values differ over time; that is, determining if the parameters are invariant across time. Longitudinal measurement invariance is not frequently considered in applied longitudinal research, instead it is just assumed (Brown, 2006). However, establishing longitudinal invariance is essential to truly understand change over time. Namely, the absence of longitudinal invariance could indicate a change in the construct over time or a change in the measurement of the construct. Clearly, the latter causes issues with drawing meaningful conclusions from a model (Nesselroade, 
1983).

Golembiewski, Billingsley, and Yeager (1976) defined three types of change: alpha, beta and gamma change. Beta change occurs when indicators being used to measure a construct do not remain consistent over time, typically due to a change in the measurement scale. Gamma change occurs when there is an actual change in the meaning of a construct over time. Finally, alpha change occurs only when both the construct and the indicators being used to measure the construct are not changing over time. Alpha change is also called the true score change; that is, true changes in the level of a construct over time. The key interest in many longitudinal studies lies in the study of true score change. In order to study whether and how true scores have changed from one time point to the next, researchers have to first exclude possibilities due to beta and gamma changes. That is exactly the goal longitudinal researchers seek to establish in searching for longitudinal invariance.

More generally, whether one is working with longitudinal or cross-sectional (e.g., multiple-group) data, two types of invariance are of concern: configural and metric. Configural invariance is established when the free and fixed parameters in a measurement model have the same patterns across time or subgroups; whereas, metric invariance occurs when the parameters are identical across time or subgroups. For example, in a longitudinal factor analysis model with multiple repeated assessments, configural invariance is established when the zero and non-zero factor loadings are located in the same positions across time; metric invariance, in contrast, is only established when the factor loadings are the same across time (Meredith, 1993; Vandenberg \& Lance, 2000). Clearly, metric invariance is a stronger form of invariance than configural invariance. If a researcher is able to establish metric invariance in a longitudinal model, he or she can more justifiably examine changes at the true score level. This is, of course, still subject to additional assumptions and restrictions (e.g., the model provides a reasonable approximation to the underlying change process 
and the indicators have satisfactory reliability, to name a few).

\subsection{Objectives for Thesis}

The overall objective of this thesis is to propose and evaluate a longitudinal MTSM model that is suited for representing the state fluctuations of affect factors over time taking into account the method in which the affects were assessed using ordinal data. The specific goals of this thesis are as follows: 1) I will evaluate and retain affect items that have "desirable" item properties using IRT for further model fitting, 2) longitudinal invariance of the selected items will be tested, 3) I will develop and evaluate a series of time series models for describing the over-time variations in the affect and method factors and 4) I will test a series of hypotheses aimed at examining the stability and changes in the method and affect factors over time. 


\section{CHAPTER 2}

\section{Methods}

\subsection{Illustrative Example}

The dataset used to illustrate the proposed longitudinal M-TSM model was part of the Affective Dynamics and Individual Differences (ADID) Study (Emotions and Dynamic Systems Laboratory, 2010). The original goal of the study was examine the dynamics of emotion regulation in a laboratory setting and to examine day-to-day variability of positive and negative affects using an experience sampling design. Affective ratings collected during the initial laboratory visit and later in an individual's everyday life will be considered as measures collected using two different "methods," namely, in laboratory setting (denoted herein as the "lab" effect) and in everyday setting (denoted herein as the "diary" effect).

\subsubsection{Participants and Measures}

A total of 271 participants ( 89 males, 182 females) were included in the study and their ages ranged from 18 to 90 years old. The participants were university students, faculty and staff as well as participants recruited from the community.

The only measure considered for this study is the Positive and Negative Affect Schedule (PANAS). The PANAS is a 20-item self-report measure with items that serve as markers of positive or negative affect (Watson, Clark, \& Tellegen, 1988). Respondents were asked to rate the extent to which they have felt each emotion on

a four-point Likert-type scale $(1=$ Never, 2 = Rarely, 3 = Often, 4 = Very Often $)$. 
The PANAS defines positive affect as pleasurable engagement and high energy and negative affect as unpleasurable engagement and distress (Watson et al., 1988). Furthermore, the PANAS provides independent measures of positive affect and negative affect; namely, they are postulated to show zero correlation based on Watson et al. (1988)'s theoretical structure of emotions.

\subsubsection{Procedures}

In a laboratory setting, participants were given a series of emotion disclosure sections to complete (neutral, positive, negative) in randomized order. All participants started with the neutral disclosure but the order of the remaining disclosure sections (negative and positive) was randomized. During the neutral disclosure, participants described pictures from the International Affective Picture System (IAPS; Lang, Bradley, \& Cuthbert, 2005), which consist of neural objects such as geometric shapes and household objects. During the positive and negative disclosures, participants described happy memories and a recent event that angered them. After each section, participants were shown a slide show of 15 negative images from the IAPS while having physiological data collected. Furthermore, after watching the slide show, participants were asked to report their momentary feelings using items from the PANAS then given a relaxation period.

A follow-up daily diary study was completed after the lab experiment. Participants were asked to provide ratings on a series of affect and personality measures,

which included the PANAS items, for 30 days (five times a day). On the survey, participants were asked to indicate the extent they felt a particular feeling or emotion since last completing the questionnaire; participants were asked to keep between an hour and a half and four hours between diary completions. For the purposes of this thesis, days two, three and four from the diary will be used. Since the PANAS items were asked five times daily, I will randomly select a time for each individual from each day. It is important to note these times will be selected to minimize the presence 
of missing data; for instance, only the non-missing occasions from a particular day will be considered.

\subsection{Model Descriptions}

\subsubsection{Preliminary Data Exploration}

The data were examined for any outliers and none were detected. The final data set was compiled using R. Randomly selected time points from days two, three and four were selected from the diary and compiled with the three time points from the lab. After listwise deletion of any missing data across all time points, the final dataset contained 235 participants.

\subsubsection{Item Exploratory Analysis}

The PANAS has multiple ordinal items that serve as indicators of positive and negative affects. Due to known convergence issues with the multitrait-multimethod models (Eid, 2000), it is advantageous for us to reduce the number of items used in model fitting. This section describes the process for item selection. Specifically, emphasis will be placed on retaining items that display desirable psychometric properties. Preliminary item-level analysis will first be performed using IRTPRO (Cai, du Toit, \& Thissen, 2011) as an initial exploration of the item characteristics.

The IRT analysis. The goal of the IRT analysis is to remove any items that have less desirable measurement properties. This is defined as items with low information and thresholds that are not well separated. In order to do this, separate univariate graded response models (Samejima, 1969) will be fit to items for each affect, method and time point, yielding a total of twelve separate models. For instance, in the first model, the graded response model will be fit to items that serve as indicators of positive affect in the lab. Information curves and trace lines will then examined to determine if any patterns exist in the same item across both methods and time points.

Figure 2.1 shows an example of a "more desirable" item and a "less desirable" 
item. The solid lines represent the trace lines; whereas, the dotted lines represent information. For the more desirable item, there is a high information curve across $\theta$ with clear peaks; conversely, for the less desirable item there is low information across a range of $\theta$ values. Thus, the less desirable item is not very informative of an individual's trait level. Secondly, the less desirable item does not have well separated trace lines. This is problematic because there is not much variation in response categories across different levels of $\theta$. Ideally, a desirable item is one with trace lines that discriminate well between categories; that is, trace lines that provide good separation across different levels of $\theta$.

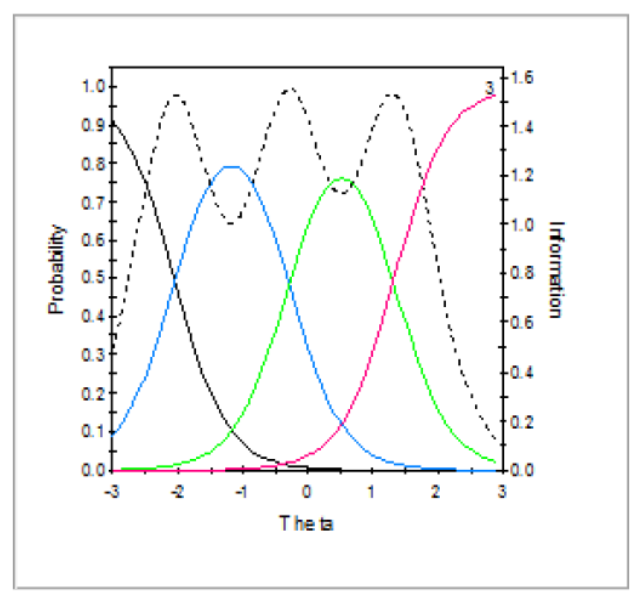

(a)

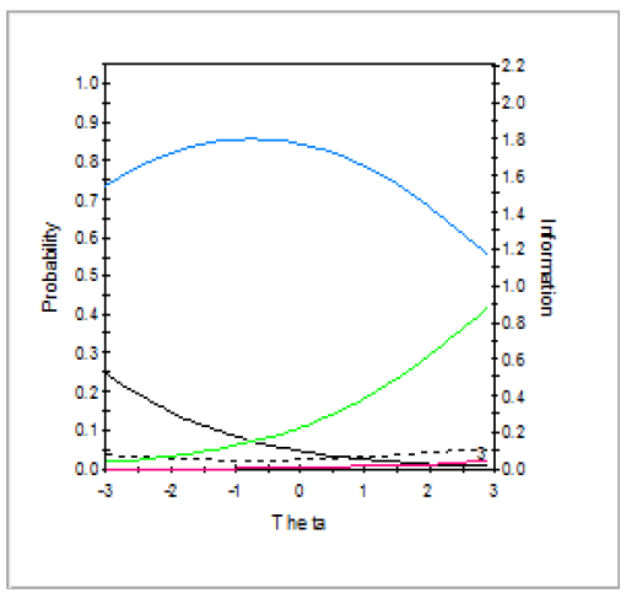

(b)

Figure 2.1: Example of (a) a"more desirable" item and (b) a "less desirable" item.

\subsubsection{Longitudinal M-TSM Model for the PANAS Data}

The proposed model consists of data from three time points. For each time point, there are four latent state factors of interest, including PA lab, PA diary, NA lab and NA diary. These state factors correspond to the participants' momentary positive affect and negative affect when assessed in a diary versus lab setting. Furthermore, it is assumed that there are higher-order shared sources of variance in the participants' positive affect and negative affect across all time points and method settings. Such 
shared sources of variances can be regarded as higher-order trait factors that capture stable interindividual differences in overall levels of PA and NA. These trait factors, denoted herein as PA trait and NA trait factors, are second-order factors that capture systematic individual differences that are shared across time as well as method settings. It is important to note that the residual variance of the state factors represents what is common to all the items at a particular occasion and not carried to the next time point. Whereas, the trait variance represents what is common to all the items across time and setting. The trait-state representation is much like the trait-state model proposed by Kenny and Zautra (2001).

The proposed model consists of a measurement model and a structural model. The measurement model for the ordinal responses is

$$
\boldsymbol{y}_{i t}^{*}=\boldsymbol{\mu}+\Lambda_{y} \eta_{i t}+\varepsilon_{i t}
$$

where $\boldsymbol{y}_{i t}^{*}$ is a $p \times 1$ vector of unobserved continuous response variables for person $i$ at time $t, \boldsymbol{\Lambda}_{y}$ is a $p \times m$ factor loading matrix, $\boldsymbol{\eta}_{i t}$ is an $m \times 1$ vector of latent endogenous variables for person $i$ at time $t$, and $\varepsilon_{i t}$ is a $p \times 1$ vector of measurement errors.

The $j$ th unobserved continuous response variables $y_{j, i t}^{*}$ is linked to the $j$ th ordinal manifest variables $y_{j, i t}$ by

$$
y_{j, i t}=k \text { if } \tau_{j, k-1}<y_{j, i t}^{*} \leq \tau_{j, k}
$$

where $\tau_{j, k}$ are threshold parameters.

Finally, the structural part of the model involves a VARMA process at the latent level. First introduced by Browne et Nesselroade (2005) with the name process factor analysis model, the process considered differs from conventional VARMA processes in that the VARMA specification is used to describe how latent factors change over 
time. The model proposed in this thesis expands the process factor analysis model by explicitly including a vector of second-order trait factors, yielding

$$
\boldsymbol{\eta}_{i t}=\boldsymbol{\Lambda}_{\eta} \text { trait }_{i}+\mathbf{A}_{1} \boldsymbol{\eta}_{i, t-1}+\mathbf{A}_{2} \boldsymbol{\eta}_{i, t-2}+\ldots+\mathbf{A}_{r} \boldsymbol{\eta}_{i, t-r}+\boldsymbol{\zeta}_{i t}
$$

where $\eta_{i t}$ is $6 \times 1$ vector of latent endogenous variables for person $i$ at time $\mathrm{t}$ consisting of the state factors: PA lab at time $t$, PA diary at time $t$, NA lab at time $t$, NA diary at time $t$, lab at time $t$ and diary at time $t$; trait $_{i}$ denotes a vector of trait factor scores for person $i$. In the present application, these trait factors include positive and negative affect at the trait level (i.e., second-order factors). $\Lambda_{\eta}$ is a factor loading matrix linking a vector of trait factors for person $i$ to the state factors of all time points. $\zeta_{i t}$ is a vector of random shocks and $\mathbf{A}_{1}, \ldots, \mathbf{A}_{r}$ are autoregressive weight matrices. The vector of second-order trait factors, trait $_{i}$, is normally distributed with zero means and variances $\sigma_{\zeta_{P}}^{2}$ and $\sigma_{\zeta_{N}}^{2}$, respectively, and covariance $\vartheta_{P N}$. The loadings of the trait factors on all state factors are constrained to be invariant across time to reflect the time-invariant nature of the trait factors.

Figure 2.2 shows a path diagram representation of the basic proposed model. This proposed model contains repeated measures (over three time points) of the positive and the negative affect items from the PANAS identified in the item-level analysis. The model shown in Figure 2.2 features three lab and three diary factors corresponding to the three repeated measurement occasions. Only the lag-one autoregression (e.g., from PAL1 to PAL2) but not the cross-regression (e.g., from PAL1 to NAL2) paths are depicted in the path diagram. Whether the cross-regression paths are needed will be evaluated as testable hypotheses. As mentioned, two trait factors, representing trait PA and trait NA, are specified to capture higher-order shared valence effects among the first-order state affect factors. For instance, trait PA was allowed to load on all the state PA factors (PAL1-PAD3) whereas trait NA was allowed 
to load on all the state NA factors (NAL1-PAD3).

One issue of interest is the specification of identification constraints for the longitudinal M-TSM model. Within the time points, each state latent variable will be specified to have the first factor loading set to one. Invariance constraints are imposed on the second-order factor loadings over time. This is because the trait factors are purported to reflect constructs that are invariant and stable across time. In addition, the factor loadings of the trait factors on the lab-based state affect factors were set to unity for identification purposes. The path diagram shown in Figure 2.2 shows a model with these constraints specified for all time points. Whether this specific set of constraints needs to be relaxed will be assessed in the model fitting process. Furthermore, due to convergence issues with MTMM models, it might be necessary to consider variations along the line of the correlated trait-correlated method minus one model (Eid, 2000). In this case, loadings on a chosen reference method would be constrained to be zero. The correlated trait-correlated uniqueness model (Kenny, 1976 ; Marsh, 1989), however, would be too restrictive for the proposed model because autoregressive paths between method factors would not be allowed. Thus, this specific variation will not be considered.

A series of models will be fit in order to determine the final M-TSM model. First, an examination of longitudinal invariance in the factor loadings and thresholds will be performed. This is important because if invariance cannot be established, that introduces added confounds and issues of change cannot be examined as readily. Furthermore, invariance in the intercepts of the state factors will also be examined. In the model shown in Figure 2.2, the intercepts of all state factors were set to zero. Deviations of these intercepts from zero may indicate the existence of over-time trends in the state factors, which could lead to spurious interpretations of other time series parameters. Thus, as part of the invariance analysis, the intercepts of the state factors at time 1 are fixed at 0 for identification purposes; other intercept terms at later 


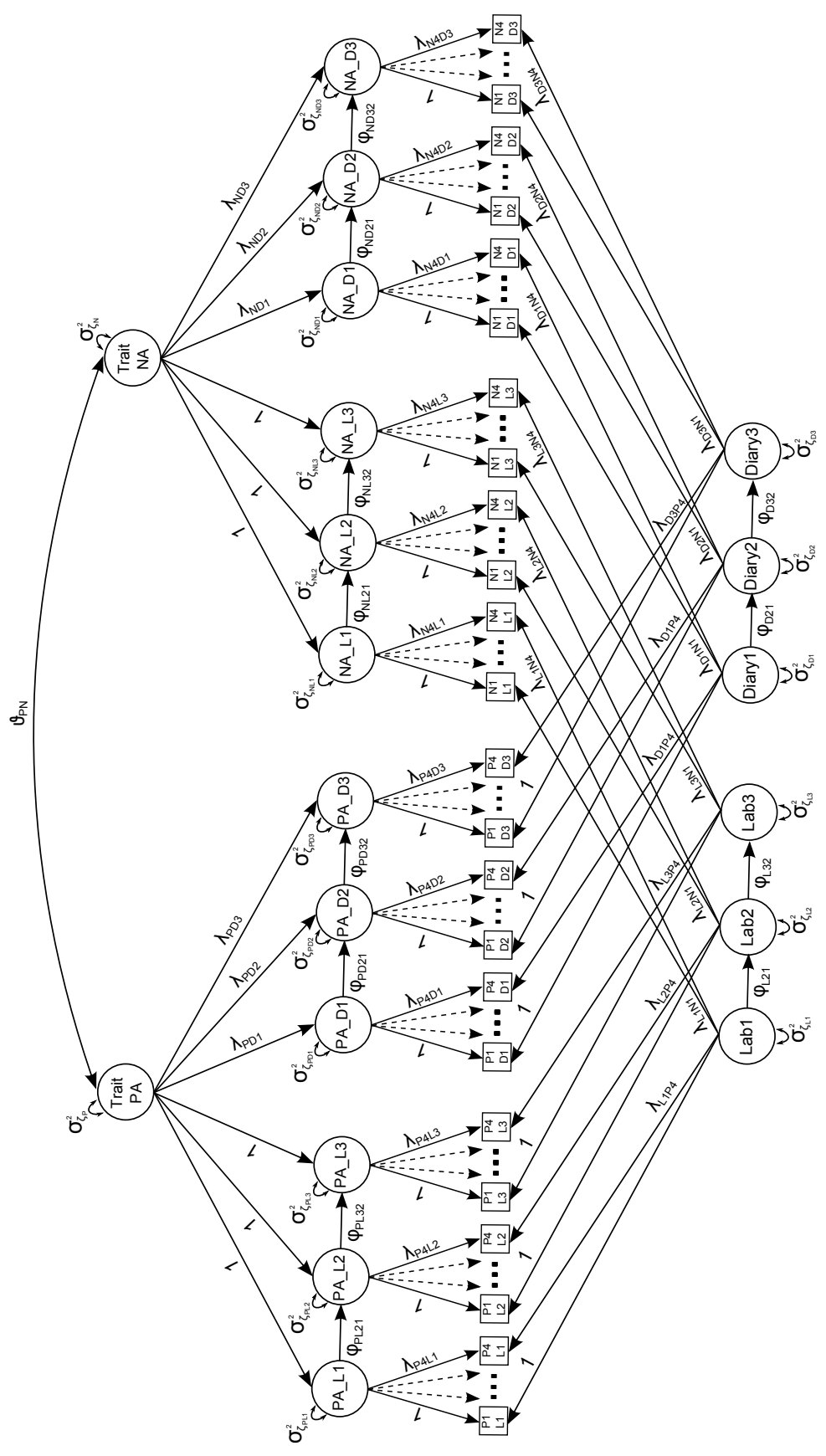

Figure 2.2: Path diagram of a longitudinal M-TSM model with VAR(1) relationships at the factor level. PA Mt $=$ Positive Affect for method $m$ at time $t, N A \_t=$ Negative Affect for method $M$ at time $t$, Labt $=$ Lab at time $t$ and Diaryt $=$ Diary at time $t$. The factor loadings for the state factors are represented by $\Lambda_{A t M t}$ where $\mathrm{A}=$ Positive or Negative Affect, $\mathrm{M}=$ Diary or Trait, and $\mathrm{t}$ is the time point. Likewise, the factor loading for the "method" factors are represented by $\lambda_{M t A t}$. The trait factor loadings are represented by $\Lambda_{A M t}$. The arrows depicted here correspond to elements in $\Lambda$ from equation 2.1, but the thresholds from equation 2.2 are not depicted directly in the path diagram. Correlations are represented as $\theta$ and autoregressive parameters as $\varphi$. 
time points will be freed to evaluate the possible existence of trends. Secondly, various models with different time series structures will be considered. Finally, perfect stability within the method factor will be tested.

Examining Longitudinal Invariance. The general procedure will be to begin with a model with the least invariance constraints (namely, the model shown in Figure 2.2 wherein all factor loadings and thresholds are freed). Two models that were nested within the first model will be then considered sequentially, with different invariance constraints imposed. A summary of these models is shown in Table 2.1. As mentioned, two types of invariance are of interest: configural and metric. The goal of the invariance analysis is to retain items that at least display configural, or preferably, metric invariance. As shown in Table 2.1, invariance constraints will be sequentially imposed on the factor loadings followed by the thresholds.

In structural equation models involving continuous data, likelihood ratio tests are often use to assess the relative fit of nested models (Bollen, 1989). However, when categorical data are used with estimation methods such as the weighted least squares means and variance adjusted (WLSMV) estimation (as will be used in the present thesis), the differences in chi-square values between nested models are not chi-square distributed. To adjust for this, the scaled chi-square difference test proposed by Satorra and colleague (Satorra \& Bentler, 1999 ; Satorra, 2000 ; Satorra \& Bentler, 2010), as implemented in the DIFFTEST option in Mplus (Asparouhov \& Muthén, 2006), is used to examine changes in fit functions between models.

Table 2.1: Models Used to Examine Longitudinal Invariance in the PANAS Items.

\begin{tabular}{|l|l|}
\hline Model 1 & Free loadings, free thresholds \\
\hline Model 2 & Invariant loadings, free thresholds \\
\hline Model 3 & Invariant loadings, invariant thresholds \\
\hline
\end{tabular}

Three different structural models will be considered. Each model will consist 
of a different specification of auto- and cross-regression structures. Autoregressive paths will be specified for both the affect and the method factors (up to a lag order, $r$, to be determined empirically by evaluating the statistical significance of high-order autoregression parameters via Wald tests) to indicate the stability of these factors over time; however, cross-regression paths will only be specified between the affect factors. The models considered are: 1) a model with no auto-regression and no crossregression (denoted as the baseline model), 2) a model with only autoregressive paths and no cross-regression and 3) a model with both auto- and cross-regression paths of the optimal order.

I will then proceed to evaluating alternative models with further simplification in the auto- and cross-regression structures. Four models will be considered for this. The models will consist of a combination of a different number of lab and diary factors. That is, the model will have either one factor representing all time points or one factor for each time point. The models considered are: 1) one lab, one diary, 2) one lab, three diary, 3) three lab, one diary and 4) three lab, three diary.

The justification for one factor verses three is testing for perfect stability. That is, if a one-factor model is chosen over a three-factor model, then there is perfectly stability over time for that factor, i.e. individuals' factor scores at a later time point are identical to their factor scores from earlier time points. Given the close proximity of time points in both the lab and diary, perfect stability should be tested. It is possible to constrain the one-factor models to be nested within the three-factor models. To illustrate this, consider a model with no trait factor and only the method factors. In this case, Model (1) is nested within Model (2); they are equivalent if the following conditions are met in Model (2): (1) longitudinal invariance constraints are imposed on the factor loadings, item thresholds, measurement error variances, (2) there are no cross-regression paths and only lag- 1 auto-regression paths are retained, (3) the lag-1 auto-regression paths connecting diary factor from time 1 and time 2 as well as 
from time 2 to time 3 are constrained to be unity, and (3) the diary factor residual variances at time 2 and time 3 are specified to be zero. Thus, likelihood ratio tests can, in principle, be performed on nested models with appropriate constraints to test the over-time stability of the method factors. However, due to the complexity of the factor structures in the proposed longitudinal M-TSM model when cross-loadings on the trait factors are present, other fit indices such as the RMSEA, CFI and TLI will be used, as appropriate, to simplify the model selection process. 


\section{CHAPTER 3}

\section{Results}

The results section is organized as follows: 1) I will discuss results from the IRT analyses, 2) results for examining longitudinal invariance will be presented, 3) time series results and model testing concerning the stability of the state factors will be discussed and 4) substantive findings and implications will be discussed in the context of the final model.

\subsection{Results for Item Analysis}

\subsubsection{IRT Analyses}

The PANAS contains a total of 20 items; however, three items were not measured in the laboratory portion of the study ("afraid," "hostile," "excited"). These items were therefore excluded in all subsequent analyses. Twelve univariate graded response models were run in order to access item properties. The primary reason for removing an item was because of low information. In other words, that item did not measure an individual's trait level on positive or negative affect very well. Issues with poor trace line separation were also found; that is, there was not enough variation in response categories (see Figure 2.1). Finally, the $\chi^{2}$ LD statistic was used to remove any items that displayed local dependence using a cutoff score of 10 (Chen \& Thissen, 1997). The final list of items retained is shown in Table 3.1. The reduced set of items was found to yield interpretable factor loading and threshold structures. Furthermore, these items also provide good coverage of the 20 content areas in which 
Table 3.1: Final List of PANAS Items Retained.

\begin{tabular}{|l|l|}
\hline Positive Affect & Negative Affect \\
\hline Active & Ashamed \\
\hline Attentive & Distressed \\
\hline Inspired & Irritable \\
\hline Strong & Jittery \\
\hline
\end{tabular}

the PANAS was based (Zevon \& Tellegen, 1982). In fact, only "active" and "strong" overlap within the same content area; therefore, these eight items cover seven of the 20 content areas.

\subsubsection{Establishing Longitudinal Invariance}

Following the steps outlined in Table 2.1, the proposed model shown in Figure 2.2 was first fit with no invariance constraints on the loadings and thresholds. To aid model convergence, I began by constraining all lag-one autoregressive parameters and the residual variances to be invariant across time. Model fit indices indicate that fit of the model was relatively poor $\left(\chi^{2}(78)=318.289\right.$, RMSEA $=0.114, C F I=0.927$ and TLI $=0.942)$. However, there was evidence suggesting configural invariance. That is, all significant loadings had the same sign and relative magnitude. Some of the residual variances were close to zero or negative and had to be constrained to be greater than zero. As a result, I did not test for metric invariance formally using a likelihood ratio test because the DIFFTEST option was not available with the modeling constraints. ${ }^{1}$ Furthermore, the correlations between positive and negative affect within lab were non-significant so those were constrained to be zero as well.

After invariance constraints were imposed on the factor loadings, a second model with time-varying thresholds was considered. The thresholds were examined and their magnitudes were found to be largely invariant across time. Figure 3.1 shows

\footnotetext{
${ }^{1}$ Models with time-varying time series parameters and time-varying residual variances were also run but there was no substantial change in conclusions.
} 
the trace lines for "irritable" across time and method. As shown for this example item, the general patterns of separation between trace lines were sustained across all three assessment points. Other items considered were also observed to show similar, largely invariant threshold structures over time.

Finally, invariance of the state factor intercepts and any potential deviations from zero (i.e., an indication of the presence of trends) were examined. To do this, the intercepts of the state affect factors at the second and third time points were allowed to be freely estimated. Because there is no theoretical reason to assume systematic trends in the method factors (lab and diary), the intercepts of the method factors were kept at zero. Results showed that all estimated intercepts were non-significant; therefore, no evidence for systematic mean change or trend was found.

The model from this section imposes invariance constraints on the factor loadings and thresholds across time. Additionally, the model assumes all intercepts to be zero across time and correlations within time were constrained to be zero between positive and negative affect for state factors.

\subsection{Most Parsimonious Model}

This subsection presents the results from examining the time series structures of the state affect factors and whether perfect stability can be established in the method factors. The latter suggests that only one lab and one diary factor (or a combination) is needed for all three time points. This indicates there is no additional uncertainty from one time point to the next in the lab and diary setting. Emphasis will be placed on interpreting the results corresponding to the final model.

To determine the order and structure of the VAR relations among the state affect factors, Wald statistics were examined and any non-significant time series parameters were set to zero. Because data were only available from three time points, the highest lag order possible was a lag of two (i.e., a VAR(2) structure). Results indicated that the significant parameters were lag-one autoregression parameters for negative affect 

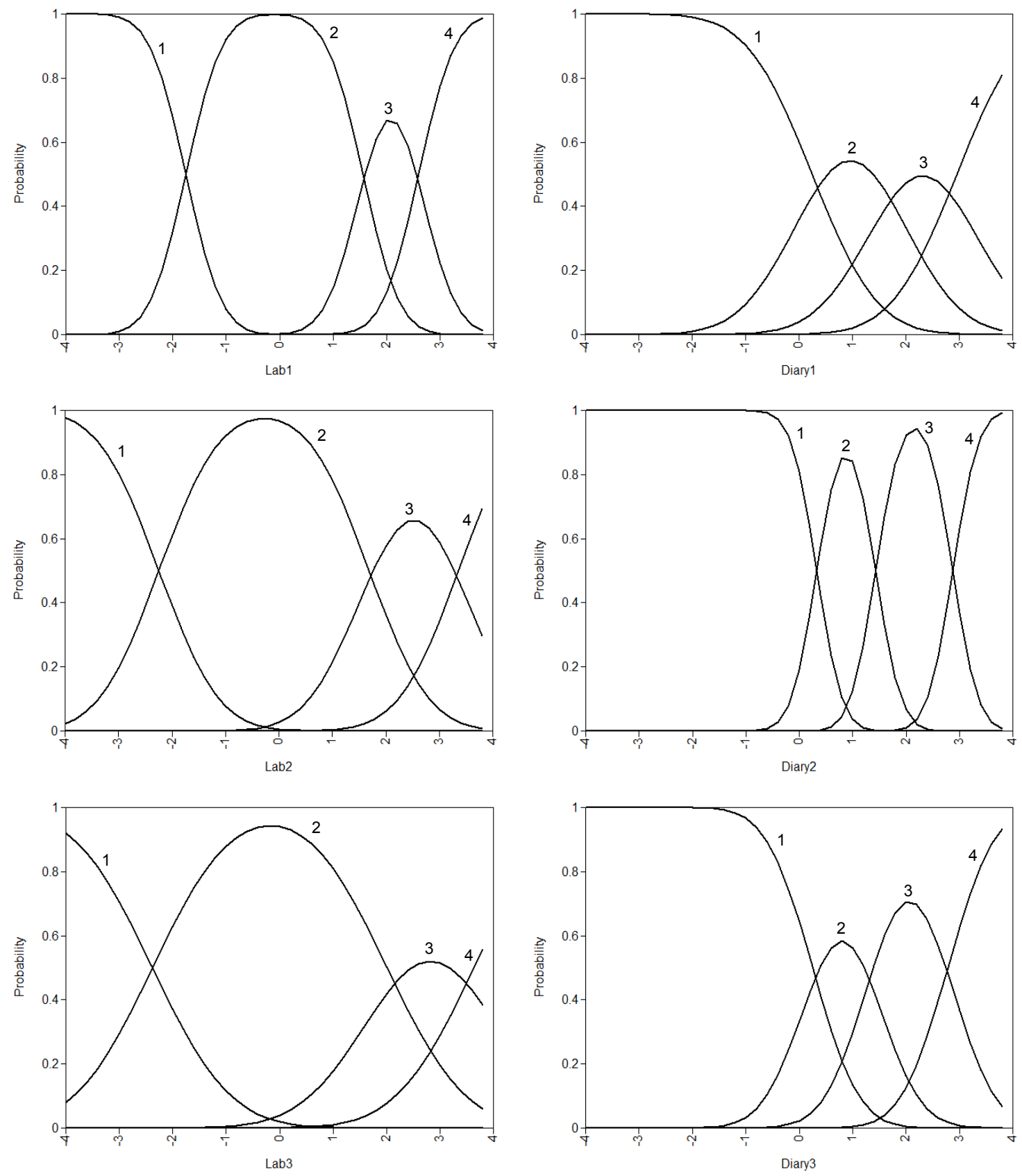

Figure 3.1: Trace lines for "irritable" over time and across method. 
in the diary and positive affect in the lab, and lag-two autoregression parameters for negative affect in the diary as well as positive affect in both the lab and diary.

Four models were fit to examine the hypothesis concerning perfect stability in the methods factor. These models were treated as non-nested; the three method factors were collapsed into one by creating one method factor. Table 3.2 shows fit indices for the four models considered. There were no substantial changes in model fit between the models so the most parsimonious model, namely, the one lab, one diary model, was chosen. Furthermore, formulating the model with fewer method factors over time as nested within the model with separate method factors at each time point led to negligible changes in model fit and no difference in conclusions.

Table 3.2: Models Used to Examine Perfect Stability in the Method Factors

\begin{tabular}{|l|l|l|l|l|}
\hline Model & RMSEA & CFI & TLI & Chi-square \\
\hline Three lab, three diary & 0.112 & 0.926 & 0.945 & $326.086(\mathrm{df}=83)$ \\
\hline Three lab, one diary & 0.111 & 0.927 & 0.945 & $324.612(\mathrm{df}=83)$ \\
\hline One lab, three diary & 0.112 & 0.927 & 0.945 & $325.855(\mathrm{df}=83)$ \\
\hline One lab, one diary & 0.112 & 0.926 & 0.945 & $326.081(\mathrm{df}=83)$ \\
\hline
\end{tabular}

Note: The degrees of freedom ( $\mathrm{dfs}$ ) for all four models were the same despite the specification of fewer factors and the omission of autoregressive parameters for the method factors in the less complicated model. This was because when WLSMV estimation is used, the df has to be estimated and not just based on the difference in the number of parameters (Muthén \& Muthén, 1998-2007).

Table 3.3 presents the parameter estimates obtained from the most parsimonious model. The most parsimonious model consists of one lab and one diary factor and all statistically significant parameters. Model fit indices indicate that fit of the final model was not great by conventional standards $\left(\chi^{2}(83)=326.081\right.$, RMSEA $=0.112$, $\mathrm{CFI}=0.926$ and TLI $=0.945)$. However, the poor fit can be understood since slight modeling discrepancies at various levels (e.g., mild violations of longitudinal invariance assumptions on the threshold parameters) can compound into large reduction in fit given the complexity of the model. In addition, there is no clear consensus on 


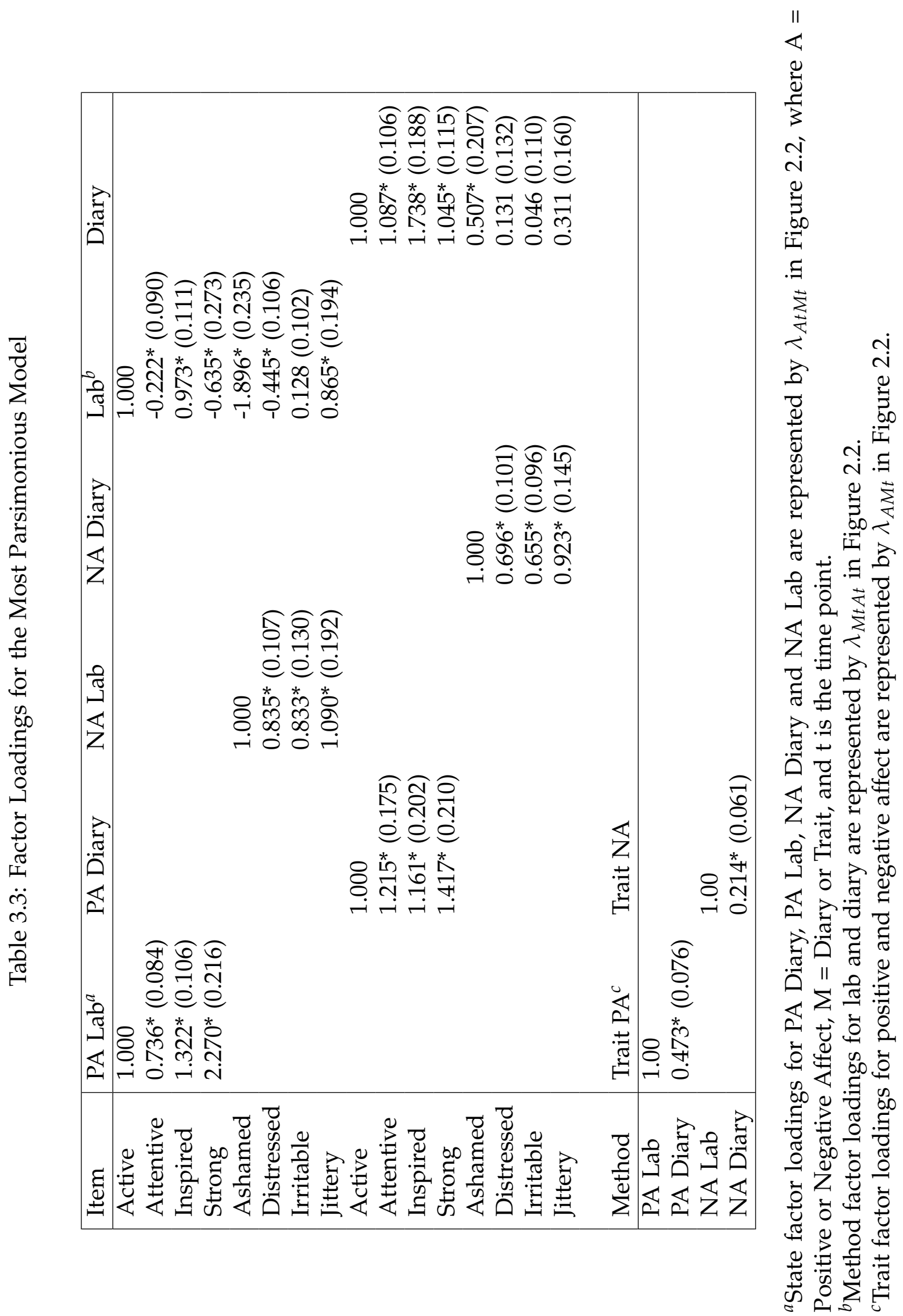


whether conventional standards for approximate good fit are applicable to models with categorical data.

The loadings for the state affect factors are all positive and high. This indicates a well-defined factor structure for the state affect factors. The factor loading patterns for the diary factor was also relatively clean. However, three of the factor loadings for negative items were not significantly different from zero in the diary factor. This suggests that these items did not contribute significantly to explaining the variance in the diary factor.

A mixed pattern of factor loadings was observed for the lab factor. The general pattern holds even when invariance constraints were not imposed on the loadings. For instance, individuals responding in higher categories of "inspired" are responding to lower categories of "attentive" and "strong." This illustrates the complexity of affective responses that arose in the lab setting. The affect induction procedures in the lab (emotion disclosure sections and negative slide shows) might have led to particularly intense and mixed affective responses that did not all show the same levels of continuity over time, as captured by the autoregressive parameters of the state affect factors. Some of these lingering affective reactions were picked up by the lab factor, leading to loadings of opposite signs between some of the PA and NA items.

As mentioned, covariances between positive and negative affect within each time point were found to be nonsignificant; whereas, the covariance between positive and negative affect at the trait level was -0.48 (corresponding to a correlation of -0.32 ). Watson, Clark and Tellegen (1988) argued that positive and negative affects are orthogonal, i.e. with a zero covariance between the two. While that was found in the state factors, the trait factors displayed a moderately negative covariance. This does not conflict with previous research as other studies have found a moderately negative covariance between positive and negative affect in the PANAS (Feldman Barrett \& Russell, 1998). The more interesting aspect is that the state factors show indepen- 
dence while the trait factors do not. One possible explanation to this is that the trait PA and trait NA are capturing low activation affective reactions, such as happinesssadness type of emotions, which Watson and Clark (1997) argued are inversely related. In contrast, the state PA and state NA might be getting at high activation affective reactions, such as excitement-anger type of emotions, which would show independence (Watson \& Clark, 1997).

Table 3.4 shows the random shock variances from the most parsimonious model. Of particular interest are the initial and residual variances for the state variables. The initial variance represents the random shock variance at the first time point; whereas, the residual variances are random shock variances after taking into account the effect of previous time points via time series parameters. For both positive and negative affect, the state factors in the diary setting have more random shock variance than in the lab setting. That is, the diary setting has more fluctuations in random shocks than the lab setting. Given the homogeneous nature of the experimental procedures implemented across the three lab occasions, less within-person fluctuations in positive and negative affects can be expected across time in the lab setting. In fact the study was designed such that participants viewed the slide show with comparable negativity ratings at each time point; therefore, not as much variability would be expected in emotions from one time point to the next. However, the emotion induction procedures could create some affective changes but such deviations might have diminished by the end of the negative slide shows.

Table 3.5 shows the time series parameter estimates for the most parsimonious model; note that negative affect within the lab setting is excluded since there were no significant autoregression parameters. The estimates of the autoregression parameters suggested that both the PA and NA state fluctuations were stationary in nature ${ }^{2}$.

\footnotetext{
${ }^{2}$ That is, the eigenvalues of the transition matrix have moduli less than one or alternatively, all the roots of the characteristic polynomial are outside the unit circle (Hamilton, 1994 ; du Toit \& Browne, 2007).
} 
Table 3.4: Random Shock Variance Estimates for the Most Parsimonious Model

\begin{tabular}{|ll|ll|}
\hline Factor & Estimate & Factor & Estimate \\
\hline TraitPA & $1.342^{*}(0.233)$ & TraitNA & $1.720^{*}(0.439)$ \\
Lab & $0.692^{*}(0.151)$ & Diary & $0.712^{*}(0.122)$ \\
PAL1 & $0.085(0.044)$ & PAL2,3 & $0.001(0.001)$ \\
PAD1 & $0.226^{*}(0.067)$ & PAD2,3 & $0.364^{*}(0.075)$ \\
NAL1 & $0.001(0.000)$ & NAL2,3 & $0.001(0.001)$ \\
NAD1 & $2.725^{*}(0.794)$ & NAD2,3 & $1.007^{*}(0.321)$ \\
\hline
\end{tabular}

In Figure 2.2 state variance estimates are represented by $\sigma_{\zeta_{A M t}}^{2}$ and trait variance estimates are represented by $\sigma_{\zeta_{A}}^{2}$ where $\mathrm{A}=$ Positive or Negative Affect, $\mathrm{M}=$ Diary or Trait, and $\mathrm{t}$ is the time point.

To help clarify the roles of these time series parameters, Figure 3.2 shows simulated trajectories of the over time dynamics generated using the estimated autoregression parameters shown in Table 3.5. These trajectories show the impact of a shock applied to the first time point and how its impact dissipates over time in the absence of any additional random shocks. As depicted, the stationary nature of the processes is evident from the return of the trajectories to a stable baseline over time.

Table 3.5: Time Series Parameters for the Most Parsimonious Model

\begin{tabular}{|lll|}
\hline Factor & Lag-one estimate $\left(\varphi_{t_{2} t_{1}}\right)$ & Lag-two estimate $\left(\varphi_{t_{3} t_{1}}\right)$ \\
\hline PA Lab & $0.296^{*}(0.053)$ & $-0.171^{*}(0.068)$ \\
PA Diary & $0.0(-)$ & $-0.285^{*}(0.106)$ \\
NA Diary & $0.659^{*}(0.073)$ & $0.254^{*}(0.106)$ \\
\hline
\end{tabular}

The lag-one autoregression parameter for PA Diary as well as lag-one and lag-two autoregression parameters for NA Lab were non-significant and, therefore, set to zero. Note that it is possible that non-significant estimates in earlier iterations may become significant again once you add other constraints.

As shown in Figure 3.2, positive affect has an oscillatory trajectory both in the lab and diary; whereas, negative affect shows exponential decay over time in the diary. This suggests that people have a more direct method of regulating negative emotions than positive emotions in real life settings. One explanation of the oscillatory nature 


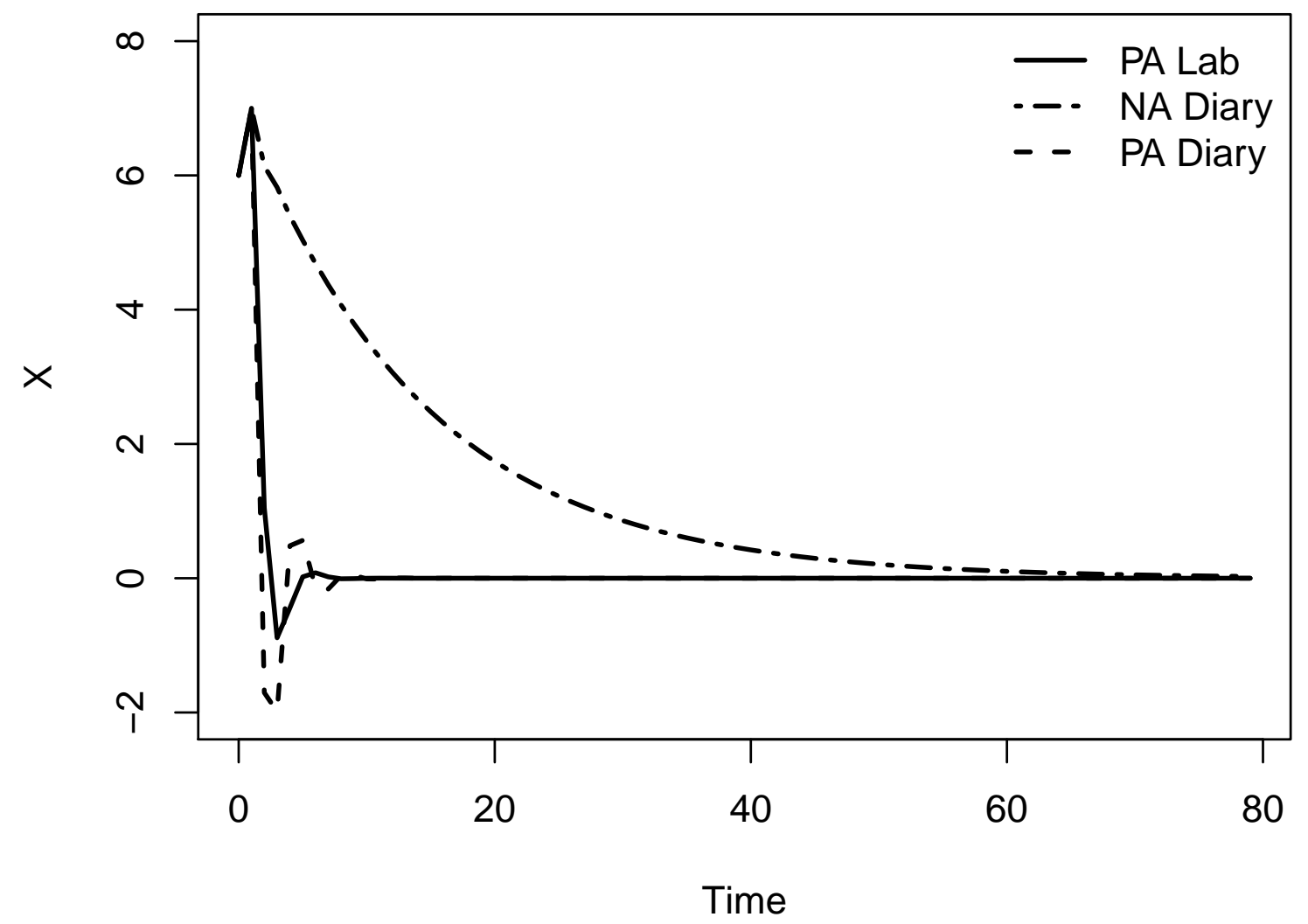

Figure 3.2: Simulated trajectories of lag-two AR processes. $\mathrm{X}$ is an arbitrary process that conforms to an AR(2) model. 
of positive affect is that when individuals are experiencing positive emotions, they are inclined not to down-regulate the positive emotions. In contrast, negative emotions constitute a less desirable state and individuals are motivated to rid themselves of such emotions.

Finally, for negative affect in the lab, previous time points do not influence negative affect at a particular time point. Given the relaxation periods between manipulations and various emotion disclosure sections in the lab setting, it is reasonable that there are no lingering effects of negative affect from one time point to another. The lab setting was designed such that each time point was a separate manipulation so it is certainly encouraging that there were no significant autoregression parameters within the lab for negative affect. Furthermore, the counterbalancing of the emotion disclosure sections in the second and third time points could have lead to a lack of significance in these parameters. Positive affect did show some continuity over time in lab, thus suggesting some differences in how individuals regulate their PA and NA over time. 


\section{CHAPTER 4}

\section{Discussion}

This thesis proposed a novel longitudinal multi-trait-state-method (M-TSM) model that combines a vector autoregressive moving average (VARMA) model at the latent level, a second-order trait factor model and a measurement model for ordinal data. Research in daily diary designs and affect data do not typically examine the convergence and discriminant validity of items asked in two different settings; instead, the items are assumed to be the same in each setting. This multi-state multimethod approach allows for the trait and state affect factors to be modeled with respect to the setting in which they were asked.

Key findings from this model show the covariance between positive and negative affect at the trait level to be moderately negative; whereas, at the state level the covariances were zero. Furthermore, time series analysis revealed that positive affect has oscillatory trajectories in both the lab and diary; whereas, negative affect shows exponential decay only in the diary setting and no lingering effects in the laboratory setting, suggesting that individuals may have a more direct method of regulating negative emotions induced by external shocks. Additionally, factor loadings on the method factors were not all significant. That is, for some of the method-specific items, no residual covariances were shared with other items after the shared covariances due to the affect factors have been extracted. A combination of positive and negative loadings was also found for the loadings of the lab-based items on the lab 
factor. This leads to difficulties in interpreting the substantive meanings of lab factor. Alternative study designs that incorporate different affect measures (e.g., self-reports and psychophysiological measures) may provide more directly interpretable meanings to the correspond method factors.

It is important to note that the ADID Study (Emotions and Dynamic Systems Laboratory, 2010) was not originally designed based on a M-TSM protocol. Some improvements could be made in study design to help answer some of the research questions that may arise in the context of longitudinal MTMM or M-TSM models. First, having more time points in the lab section would have been beneficial since this study was only limited to three time points; the diary had many more. However, the model could have certainly been formulated to include more time points from the diary. The inclusion of more time points in the lab would have led to richer findings and more in-depth evaluation of higher order autoregression structures. Second, having a more homogeneous set of stimuli in the lab and in the diary setting would have been beneficial as well. For example, participants in the lab and diary could have been exposed to stimuli designed specifically to illicit a particular type of emotion and these would have helped improve the convergence validity of the trait/state affect factors within and across time points.

The two primary features of this model include using time series techniques to capture the state fluctuations in affect over time as well as an ordinal measurement model to handle ordinal items. Specification of an ordinal measurement model is important when using ordinal data; treating these data as continuous could lead to spurious results when the categorical data show substantial deviations from normality.

Other structural models could be chosen to represent the dynamics of the system. This model used a vector autoregressive moving average (VARMA) model to handle the fluctuations in affect over time. Alternatively, if there is a growth process 
involved, then a latent growth curve model might be more appropriate as adopted in much of the prior research on longitudinal MTMM (Grimm et al., 2009; Cole et al., 1996). However, most conventional growth curve models do not provide the possibility of testing the directionality of lagged influences between processes (e.g., as captured by the cross-regression parameters). Future research could also examine alternative specifications of time series structures for the latent state processes. For this thesis, moving average parameters were not included. Inclusion of moving average components in future studies should be considered as theory justifies.

Future directions of this research topic should include the addition of covariates and the examination of differences in the dynamics of the model with regards to gender, ethnicity and such factors (Lim \& Ployhart, 2006; Kuppens, Grietens, Onghena, \& Michiels, 2009; Cole et al., 2009). For instance, gender differences in emotion regulation is not well understood (McRae, Ochsner, Mauss, Gabrieli, \& Gross, 2008); thus, it would be beneficial to answer the question: do men and women regulate emotions in the same fashion taking into account the setting in which items were asked? Such research questions can be tested by evaluating multiple-group extensions of the proposed model. Furthermore, alternative measures of positive and negative affect could be considered as well as various mood induction procedures for both the lab and diary. This might help clarify the lack of significance found in the time series parameters for negative affect in the lab.

Numerical difficulties often arise in fitting MTMM or, in the present context, MTSM models due to their general complexity. In the proposed model, including a trait component allowed us to test the assumption that state affect factors assessed using different methods were related. Less convergence issues were actually observed after the trait factors were added. Furthermore, it proved to be fairly difficult to specify an ordinal measurement model. While the process is relatively straightforward, many model evaluations features are not available with ordinal data; namely, the use of 
DIFFTEST to perform a LRT. When model constraints were specified, a LRT was not possible for the nested models. This was a major limitation in the analyses. One way to circumvent this would have been to remove all the model constraints or to resort to a continuous measurement model. For instance, item parceling (Cattell, 1956) may be used to group the items into item parcels to create continuous manifest variables.

Several methodological issues arose when designing and testing the proposed M-TSM model. The first issue concerned the time scales of the state affect factors. That is, the lab and diary are on two different time scales. Items in the lab were asked within a few hours; whereas, items in the diary were asked over three consecutive days. To account for this, a state factor for each affect in each setting for each time point was specified. This modification of the correlated trait-correlated method model (Jöreskog, 1974b; Widaman, 1985) allowed for the retention of the temporal relation between the factors while accounting for the fact that lab and diary changes unfold over two different time scales.

Second, issues with determining how to identify the model arose. This model was identified by setting the first factor loading to one for each of the state latent variables, which is a common method for identifying the metrics of the underlying latent variables (Bollen, 1989). The limitation here is that it is assumed that the corresponding item with a factor loading of one is invariant across time, which may or may not be the case. However, preliminary item response analyses showed the trace lines of all items to be stable across time; therefore, this assumption appeared tenable. Furthermore, the trait latent variable was defined explicitly by setting all of the factor loadings of the trait factors on the diary items to unity, and the factor loadings on the lab items to be invariant over time. This was done since trait factors are postulated as stable processes that remain more or less constant over time.

Finally, future models could use different specifications of the multi-trait multimethod models. That is, the correlated-trait correlated-method minus one model 
would be suited for a M-TSM model. Fortunately, convergence issues were minimal but adding in covariates and more time points could lead to issues with convergence; the correlated-trait correlated-method minus one model might help aid convergence. In the current illustrated example a reference method, such as the diary setting, could be chosen. Diary lends itself to be a reference method because it would be of interest to see if measuring individuals in a lab setting changes how they respond to items versus in a naturalistic environment.

In recent years, many alternative models have been proposed to help distinguish between trait and state processes (Kenny \& Zautra, 1995, 2001). The longitudinal M-TSM model proposed in this thesis allows the trait-state distinction to be defined using multiple assessment methods. This novel approach gives researchers greater flexibility in consolidating different emotion assessment methods over time before deriving a general, trait-based structure of emotions that remains invariant over time and settings. The union of the previous trait-state models and multi-trait multimethods models can help provide new insights into important research questions pertaining to how emotions change or remain constant in various contexts. 


\section{Références}

Andrews, F. (1984). Construct validity and error components of survey measures. Public Opinion Quarterly, 48, 409-442.

Asparouhov, T., \& Muthén, B. (2006, May). Mplus web notes: No. 10. (Robust Chi Square Difference Testing with Mean and Variance Adjusted Test Statistics)

Bagozzi, R. P., \& Yi, Y. (1991). Multitrait-multimethod matrices in consumer research. Journal of Consumer Psychology, 17, 426-439.

Bollen, K. A. (1989). Structural equations with latent variables. New York: Wiley.

Bollen, K. A., \& Curran, P. J. (2004). Autoregressive latent trajectory (alt) models: A synthesis of two traditions. Sociological methods and research, 32(3), 336-383.

Brown, T. (2006). Confirmatory factor analysis for applied researchers. New York: The Guilford Press.

Browne, M. W., \& Nesselroade, J. R. (2005). Advances in psychometrics: A festschrift for roderick p. mcdonald. In A. Maydeu-Olivares \& J. J. McArdle (Eds.), (p. 415452). Mahwah, NJ: Erlbaum.

Burns, G. L., Walsh, J. A., \& Gomez, R. (2003). Convergent and discriminant validity of trait and source effects in adhd-inattention and hyperactivity/impulsivity measures across a 3-month interval. Journal of Abnormal Child Psychology, 15, 529-541.

Cai, L., du Toit, S. H. C., \& Thissen, D. (2011). Irtpro: Flexible, multidimensional, multiple categorical IRT modeling (1st éd.). Chicago, IL : Scientific Software International. 
Campbell, D. T., \& Fiske, D. W. (1959). Convergent and discriminant validation by the multitrait-multimethod matrix. Psychological Bulletin, 56, 81-104.

Cattell, R. B. (1956). Validation and intensification of the sixteen personality factor questionnaire. Journal of Clinical Psychology, 12, 205-214.

Chatfield, C. (2004). The analysis of time series: An introduction (6th éd.). Boca Raton, FL : Chapman \& Hall/CRC.

Chen, W.-H., \& Thissen, D. (1997). Local dependence indexes for item pairs using item response theory. Journal of Educational and Behavorial Statistics, 22(3), 265289.

Cole, D., Cho, S., Martin, J., Seroczynski, A., Tram, J., \& Hoffman, K. (2009). Effects of validity and bias on gender differences in the appraisal of children's competence: Results of mtmm analyses in a longitudinal investigation. Structural Equation Modeling: A Multidisciplinary Journal, 8, 84-107.

Cole, D., Martin, J., Powers, B., \& Trugilo, R. (1996). Modeling causal relations between academic and social competence and depression: A multitraitmultimethod longitudinal study of children. Journal of Abnormal Psychology, 105(2), 258-270.

Conley, J. (1985). Longitudinal stability of personality traits: A multitraitmultimethod-multioccasion analysis. Journal of Personality and Social Psychology, 49(5), 1266-1282.

Cudeck, R. (1985). Multiplicative models and mtmm matrices. Journal of Educational Statistics, 13, 131-147.

DiStefano, C. (2002). The impact of categorization with confirmatory factor analysis. Structural Equation Modeling, 9, 327-346. 
du Toit, S. H. C., \& Browne, M. W. (2007). Structural equation modeling of multivariate time series. Multivariate Behavioral Research, 42, 67-101.

Eid, M. (2000). A multitrait-multimethod model with minimal assumptions. Psychometrika, 65, 241-261.

Emotions and Dynamic Systems Laboratory. (2010). The affective dynamics and individual differences (adid) study: Developing non-stationary and network-based methods for modeling the perception and physiology of emotions. (Unpublished manual, University of North Carolina at Chapel Hill)

Feldman Barrett, L. A., \& Russell, J. A. (1998). Independence and bipolarity in the structure of current affect. Journal of Personality and Social Psychology, 74, 967-984.

Geiser, C., Eid, M., Nussbeck, F., Courvoisier, D., \& Cole, D. (2010). Analyzing true change in longitudinal multitrait-multimethod studies: Application of a multimethod change model to depression and anxiety in children. Developmental Psychology, 46(1), 29-45.

Golembiewski, R., Billingsley, K., \& Yeager, S. (1976). Measuring change and persistence in human affairs: Types of change generated by od designs. Journal of Applied Behavioral Science, 12, 133-157.

Grimm, K., Pianta, R., \& Konold, T. (2009). Longitudinal multitrait-multimethod models for developmental research. Multivariate Behavorial Research, 44, 233-258.

Hamilton, J. D. (1994). Time series analysis. Princeton, NJ : Princeton University Press.

Iida, M., Shrout, P. E., Laurenceau, J., \& Bolger, N. (in press). Using diary methods in psychological research. In H. Cooper, P. Camic, D. Long, A. T. Panter, D. Rindskopf, \& K. Sher (Eds.), Apa handbooks in psychology: Apa handbook of research methods in psychology. Washington, DC : American Psychological Association. 
Jöreskog, K. G. (1973). A general method for estimating a linear structural equation system. In A. S. Goldberger \& O. D. Duncan (Eds.), Structural equation models in the social sciences (pp. 85-112). New York : Seminar Press.

Jöreskog, K. G. (1974a). Analyzing psychological data by structural analysis of covariance matrices. In D. H. Krantz, R. C. Atkinson, R. L. Duncan, \& P. Suppes (Eds.), Contemporary developments in mathematical psychology (vol. 2): Measurement, psychophysics and neural information processing (pp. 1-56). San Francisco : W. H. Freeman and Company.

Jöreskog, K. G. (1974b). Analyzing psychological data by structural analysis of covariance matrices. In R. L. R. Atkinson D. Krantz \& P. Suppes (Eds.), (p. 1-56). San Francisco: W. H. Freeman.

Kenny, D. A. (1976). An empirical application of confirmatory factor analysis to the multitrait-multimethod matrix. Journal of Experimental and Social Psychology, 12, 247-252.

Kenny, D. A., \& Zautra, A. (1995). The trait-state-error model for multiwave data. Journal of Consulting and Clinical Psychology, 63, 52-59.

Kenny, D. A., \& Zautra, A. (2001). New methods for the analysis of change: Decade of behavior. In L. M. Collins \& A. G. Sayer (Eds.), (p. 243-263). Washington, DC: American Psychological Association.

Kuppens, S., Grietens, H., Onghena, P., \& Michiels, D. (2009). A longitudinal study of childhood social behaviour: Inter-informant agreement, inter-context agreement, and social preference linkages. Journal of Social and Personal Relationships, 26, 769792.

Lang, P. J., Bradley, M. M., \& Cuthbert, B. N. (2005). International affective picture system (IAPS): Instruction manual and affective ratings. 
(Technical Report A-6, The Center for Research in Psychophysiology, University of Florida.)

Lim, B. C., \& Ployhart, R. E. (2006). Assessing the convergent and discriminant validity of goldbergs international personality item pool: A multitrait-multimethod examination. Organizational Research Methods, 9, 29-54.

Lord, F. (1980). Applications of item response theory to practical testing problems. Hillsdale, NJ: Lawrence Erlbaum Associates.

Luzzo, D. (1993). A multi-trait, multi-method analysis of three career development measures. The Career development quarterly, 41(4), 367-375.

Marsh, H. W. (1989). Confirmatory factor analyses of multitrait-multimethod data: Many problems and a few solutions. Applied Psychological Measurement, 13, 335361.

Marsh, H. W., \& Bailey, M. (1991). Confirmatory factor analyses of multitraitmultimethod data: A comparison of alternative models. Applied Psychological Measurement, 15, 47-70.

Marsh, H. W., \& Grayson, D. A. (1995). Structural equation modeling: Concepts, issues, and applications. In R. H. Hoyle (Ed.), (p. 177-198). Thousands Oaks, CA: Sage.

McArdle, J. J., \& Hamagami, F. (2001a). Latent difference score structural models for linear dynamic analyses with incomplete longitudinal data. In L. M. Collins \& A. G. Sayer (Eds.), (p. 137-176). Washington, DC: American Psychological Association.

McArdle, J. J., \& Hamagami, F. (2001b). Latent difference score structural models for linear dynamic analysis with incomplete longitudinal data. In L. Collins \& 
A. Sayer (Eds.), New methods for the analysis of change (pp. 139-175). Washington, D.C. : American Psychological Association.

McRae, K., Ochsner, K., Mauss, I., Gabrieli, J., \& Gross, J. (2008). Gender differences in emotion regulation: An fmri study of cognitive reappraisal. Group Processes and Intergroup Relations, 11(2), 143-162.

Meredith, W. (1993). Measurement invariance, factor analysis and factor invariance. Psychometrika, 58, 525-543.

Muthén, L. K., \& Muthén, B. O. (1998-2007). Mplus user's guide (5th éd.). Los Angeles, CA : Muthén \& Muthén.

Nesselroade, J. R. (1983). Temporal selection and factor invariance in the study of development and change. In P. B. Baltes \& O. G. Brim (Eds.), Life-span development and behavior (Vol. 5, pp. 59-87). New York : Academic Press.

Nesselroade, J. R., \& Bartsch, T. W. (1977). Multivariate perspectives on the construct validity of the trait-state distinction. In R. B. Cattell \& R. M. Dreger (Eds.), Handbook of modern personality theory (pp. 221-238). Baton Rouge, LA : Hemisphere Publications.

Samejima, F. (1969). Estimation of latent ability using a response pattern of graded scores (psychometric monograph no. 17).

Satorra, A. (2000). Innovations in multivariate statistical analysis: A festschrift for heinz neudecker. In D. Heijmans, D. Pollock, \& A. Satorra (Eds.), (p. 233-247). Kluwer Academic Publishers, Dordrecht.

Satorra, A., \& Bentler, P. (1999). A scaled difference chi-square test statistic for moment structure analysis. (Rapport technique). Technical Report University of California, Los Angeles. 
Satorra, A., \& Bentler, P. (2010). Ensuring positiveness of the scaled difference chisquare test statistic. Psychometrika, 75, 243-248.

Scherpenzeel, A., \& Saris, W. (2007). Longitundinal models in the behavorial and related sciences. In K. van Montfort, J. Oud, \& A. Satorra (Eds.), (p. 381-402). Mahwah, NJ: Erlbaum.

Schmitt, N., \& Stults, D. (1986). Methodolgical review: Analysis of mtmm matricies. Applied Psychological Measurement, 10, 1-22.

Takane, Y., \& de Leeuw, J. (1987). On the relationship between item response theory and factor analysis of discretized variables. Psychometrika, 52, 393-408.

Vandenberg, R. J., \& Lance, C. E. (2000). A review and synthesis of the measurements invariance literature: Suggestions, practices, and recommendations for organizational research. Organizational Research Methods, 3, 4-69.

Waksman, S. (1978). An application of a multitrait-multimethod test to validity data of a social learning treatment for aggressive children. Journal of Abnormal Child Psychology, 6(1), 1-10.

Watson, D., \& Clark, L. A. (1997). The measurement and mismeasurement of mood: Recurrent and emergent issues. Journal of Personality Assessment, 86, 267-296.

Watson, D., Clark, L. A., \& Tellegen, A. (1988). Development and validation of brief measures of positive and negative affect: The PANAS scale. Journal of Personality and Social Psychology, 54(6), 1063-1070.

Wei, W. W. S. (1990). Time series analysis. Redwood City, CA : Addison-Wesley.

Werts, C., Jöreskog, K., \& Linn, R. (1972). A multitrait-multimethod model for studying growth. Educational and Psychological Measurement, 32, 655-678. 
Widaman, K. F. (1985). Hierarchically nested covariance structure models for multitrait-multimethod data. Applied Psychological Measurement, 9(1), 1-26.

Willett, J. B. (1989). Some results on reliability for the longitudinal measurement of change: Implications for the design of studies of individual growth. Educational and Psychological Measurement, 49, 587-602.

Winne, P., \& Marx, R. (1977). A multitrait-multimethod study of three self-concept inventories. Child Development, 48(3), 893-901.

Wirth, R., \& Edwards, M. (2007). Item factor analysis: Current approaches and future directions. Psychological Methods, 12(1), 58-79.

Zevon, M. A., \& Tellegen, A. (1982). The structure of mood change: An idiographic/nomothetic analysis. Journal of Personality and Social Psychology, 43, 111122. 